\title{
Cópulas Dinâmicas de Lévy e suas Aplicações no Apreçamento de Opções Multidimensionais com Dependência na Trajetória
}

\author{
Edson Bastos e Santos* \\ Nelson Ithiro Tanaka**
}

\section{Resumo}

Este artigo apresenta uma alternativa para modelar opções multidimensionais, cujas estruturas de ganhos e perdas dependam das trajetórias dos processos dos preços dos ativos objetos. A modelagem sugerida considera os processos de Lévy, uma classe de processos estocásticos bastante ampla, que permite a existência de saltos (descontinuidades) no processo dos preços dos ativos financeiros, e tem como caso particular o movimento browniano. Para descrever a dependência entre os processos, os conceitos estáticos de cópulas ordinárias são estendidos para o contexto dos processos de Lévy, levando em consideração a medida de Lévy, que caracteriza o comportamento dos saltos. São realizados estudos comparativos entre as cópulas dinâmicas de Clayton e de Frank, no apreçamento dos contratos derivativos do tipo asiático, utilizando-se processos gama e técnicas de simulação de Monte Carlo.

Palavras-chave: processos de Lévy, cópulas dinâmicas, opções multidimensionais, dependência na trajetória.

Códigos JEL: C02, C13, C63, G12.

\section{Abstract}

This article presents an alternative to modeling multidimensional options, where the payoffs depend on the paths of the trajectories of the underlying-asset prices. The proposed technique considers Lévy processes, a very ample class of stochastic processes that allows the existence of jumps (discontinuities) in the price process of financial assets, and as a particular case, comprises the Brownian motion. To describe the dependence among Lévy processes, extending the static concepts of the ordinary copulas to the Lévy processes

Submetido em Novembro de 2007. Aceito em Maio de 2008. O artigo foi avaliado segundo o processo de duplo anonimato além se de ser avaliado pelos editores. Editor principal: Ricardo $\mathrm{P}$. C. Leal. Gostaríamos de agradecer ao pareceristas anônimos pelas suas valiosas recomendações. O primeiro autor agradece ao Prof. Dr. José de Oliveira Siqueira, orientador da dissertação de mestrado do respectivo autor, que originou este artigo, e ao Banco Central do Brasil pelo apoio financeiro, e observa que as opiniões apresentadas neste artigo não refletem de maneira alguma as opiniões do Banco Central do Brasil. O segundo autor agradece ao apoio parcial da FAPESP através do Projeto Temático - Processo 2004/015304-6

*Doutorando do Departamento de Administração da Faculadade de Economia, Administração e Contabilidade da USP. E-mail: edsonbastos@usp.br

**Professor do Departamento de Estatística do Instituto de Matemática e Estatística da USP. Email: nitanaka@ime.usp.br 
context, considering the Lévy measure, which characterizes the jumps behavior of these processes. A comparison between the Clayton and the Frank dynamic copulas and their impact in asset pricing of Asian type derivatives contracts is studied, considering gamma processes and Monte Carlo simulation procedures.

Keywords: Lévy processes, dynamic copulas, multidimensional options, path-dependent.

\section{Introdução}

Não é difícil se convencer que modelagem multivariada de alguns fenômenos financeiros é muito mais promissora do que considerar modelagem univariada de seus componentes separadamente. Precificação e hedging de uma cesta de opções, otimização de carteiras, simulação de cenários de riscos, etc. certamente ganham realismo na modelagem se as dependências entre os seus componentes forem levadas em consideração. Se além disso, a possibilidade de saltos nos processos envolvidos for levada em conta então mais um passo estará sendo dado em direção a modelos mais próximos das realizações observadas no mercado. A ocorrência de saltos em processos de log retornos, por exemplo, seria contemplada nesse modelo. A contrapartida é que modelos multidimensionais são mais difíceis de serem construidos e manejados que os univariados.

Uma das maneiras mais simples de modelar fenômenos multivariados é usar modelos gaussianos, onde a estrutura de dependência pode ser parametrizada em termos da matriz de correlações. Neste caso particular, as propriedades marginais, que são em parte caracterizadas pelas volatilidades, podem ser distinguidas das propriedades de dependência, que são dadas pela matriz de correlação. Outra razão para a utilização de modelos gaussianos é a possibilidade de simular séries temporais multivariadas gaussianas com matrizes de correlação arbitrárias.

No que concerne a introdução de saltos em um modelo multidimensional podemos modificar o tempo de um Movimento Browniano (MB) multidimensional com um subordinador univariado (Eberlein, 2001, Prause, 1999). Suponha que a escala temporal do mercado é modelada por um subordinador $Z=\left\{Z_{t}\right\}_{t \geq 0}$ (i.e., um processo de Lévy com trajetórias quase certamente crescentes), com expoente laplaciano $l(u), B=\left\{B_{t}\right\}_{t>0}$ é um MB $d$-dimensional com matriz de covariâncias $\boldsymbol{\Sigma}$ e médias $\mu \in \mathbb{R}^{\mathbf{d}}$. Ao modificar a escala temporal de um MB com tendência determinística $\mu$ por meio de $Z$, obtém-se um novo processo de Lévy $d$-dimensional $X_{t}=B\left(Z_{t}\right)+\mu Z_{t}$. Esta abordagem permite construir e simular modelos multidimensionais, tais como os processos Variância Gama (vide Madan et alii (1990), Madan et alii (1998) e Luciano e Schoutens (2006)), Normal, Gaussiana Invertida (vide Barndorff-Nielsen $(1997,1998)$ ) e Hiperbólico Generalizado (vide Prause (1999), Fajardo e Farias (2004, 2007)). Por outro lado, a modelagem da estrutura de dependência nesta construção mostra-se um pouco rígida, pois, apenas distibuições marginais de um mesmo mesmo tipo podem ser utilizadas. 
Para modelos de atividade finita, como o de Merton (1976), a estrutura de dependência pode ser obtida estabelecendo dependências entre os saltos individuais através de processos de Poisson unidimensionais, vide Lindskog e McNeil (2001). Se os saltos forem normais, a dependência pode ser estabelecida pela matriz de correlação. Se os saltos não forem normais, e.g., se assumirem distribuição de Laplace assimétrica, como no modelo de (Kou (2002), Kou e Wang (2004)), então, a dependência pode ser modelada por cópulas. Naturalmente, esta abordagem só faz sentido quando a quantidade de fontes de riscos que produzem os saltos é pequena, por exemplo, quando todos os componentes (processos de preços dos ativos ativos financeiros) do modelo apresentam saltos no mesmo instante de tempo. Caso contrário, esta abordagem não é parcimoniosa, levando em conta uma quantidade muito grande de parâmetros. Outra limitação desta abordagem, é que todos os componentes do modelo devem ser processos compostos de Poisson.

Ainda, encontramos outros enfoques de modelagens em Finanças usando Processos de Lévy multivariados dos quais mencionamos dois. O primeiro (vide $\mathrm{Fa}$ jardo e Mordecki (2006)) lida com o processo bivariado reduzido, usando o Teorema de Girsanov, para um mercado auxiliar que os autores denominaram de dual. O segundo (vide Matache et alii (2004)) trata de log-retornos de contratos do tipo europeu, onde a dinâmica dos processos de Lévy satisfazem equações integrodiferenciais parciais parabóliças (PIDE).

Juntando as noções acima, um modelo multidimensional apropriado, usando o Processo de Lévy, deve apresentar as seguintes propriedades: Em primeiro lugar, deve haver liberdade na especificação da lei de qualquer componente individual. Em segundo, o leque de possibilidades de estruturas de dependência deve ser amplo, incluindo dependência perfeita que também chamaremos de completa e independência e que a transição entre estes dois extremos seja harmoniosa. Por último, a parametrização deve ser parcimoniosa, ou seja, com poucos parâmetros.

Estas características podem ser obtidas se a dependência for modelada de maneira independente do comportamento marginal dos componentes, i.e., a modelagem da estrutura de dependência pode ser efetuada de maneira separada das distribuições marginais, por meio de cópulas. Apesar deste procedimento ser algo relativamente recente, dois livros que tratam sobre o assunto são: Nelsen (1999) e Joe (1997). Estas duas referências consideram variáveis aleatórias em apenas um determinado instante de tempo, sendo que é necessário estender estes conceitos para o ambiente dinâmico dos processos de Lévy, que é a proposta primordial neste estudo. A dependência entre os componentes de um processo de Lévy multidimensional pode ser completamente caracterizada por uma cópula de Lévy, i.e., uma função que possui as mesmas propriedade de uma cópula ordinária, entretanto, comportando as devidas modificações convenientes. Este procedimento fornece as ferramentas necessárias para construir processos de Lévy multidimensionais com dependências específicas. Existem várias famílias paramétricas de cópulas de Lévy que podem ser utilizadas para construir processos de Lévy de dimensão $n$, ou seja, podemos integrar $n$ processos de Lévy unidimensionais por 
meio de uma cópula parametrizada. No entanto, apesar dos modelos construídos por meio da abordagem cópula de Lévy descreverem todas as características de dependência multidimensional, eles podem se tornar matematicamente complexos e difíceis de serem computacionalmente implementados.

Para ilustrar a necessidade da cópula dinâmica podemos pensar que no caso de opções européias, no único horizonte temporal de exercício a dependência pode ser descrita através da cópula ordinária. Para outros tipos de opções (americana, com barreiras, exótica) há a necessidade da modelagem dinâmica da dependência nos vários instantes possíveis de exercícios, demandando o uso de cópulas de processos (no caso, de Lévy).

Apresentamos em seguida os resultados conhecidos sobre processos de Lévy. As provas dos resultados neste trabalho serão fornecidas apenas quando elas forem curtas ou apresentarem noções úteis à outros pontos do trabalho. Para as demais provas serão indicadas referências. Na Seção 2 recordamos conceitos provenientes das cópulas ordinárias. Na Seção 3, introduzimos a cópula de Lévy, que, em algumas vezes, denominamos cópula dinâmica. Na Seção 4, apresentamos generalizações dos resultados apresentados na Seção 3, e, por meio do Exemplo 29, propomos uma família de cópulas de Lévy, que denominamos Família de Frank Generalizada, dadas as suas similaridades às cópulas ordinárias de Frank. $\mathrm{Na}$ Seção 5 apresentamos exemplos de ativos financeiros e desenvolvemos a estrutura de inferência que está associada aos processos de Lévy multidimensionais. Fazemos um estudo de apreçamento de opções asiáticas através de simulação onde o modelo de base é composto por processos de Lévy e a estrutura de dependência dada pela cópula dinâmica de Lévy. Nesta seção estão concentradas as contribuições originais do trabalho. Na Seção 6, concluímos sobre os resultados obtidos no artigo, onde propomos uma extensão do Tau de Kendall para medir dependências não lineares entre processos de Lévy, resultado este que será explorado em trabalhos posteriores.

\subsection{Breve introdução aos processos de Lévy}

São apresentados algumas definições e alguns resultados sobre o Processo de Lévy que serão utilizados no desenvolvimento a seguir. Para mais detalhes e provas de resultados indicamos Cont e Tankov (2004) e Kallsen e Tankov (2006).

Um processo $\left\{X_{t}\right\}_{t \geq 0}$ em $\mathbb{R}^{d} \operatorname{com} P\left(X_{0}=0\right)=1$ é denominado ser de Lévy se:

1. possui incrementos independentes, i.e., para toda sequência de tempos $t_{0}$, $t_{1}, \ldots, t_{n}$ as variáveis aleatórias $X_{t_{0}}, X_{t_{1}}-X_{t_{0}}, \ldots, X_{t_{n}}-X_{t_{n-1}}$ são independentes.

2. os incrementos são estacionários, i.e., a lei de $X_{t+h}-X_{t}$ não depende de $t$. Isto significa que os parâmetros são constantes.

3. tem continuidade estocástica: $\forall \varepsilon>0$, 


$$
\lim _{h \rightarrow 0} \mathbb{P}\left(\left|X_{t+h}-X_{t}\right| \geq \varepsilon\right)=0
$$

onde |.| denota a norma euclidiana.

A terceira condição não implica em trajetórias amostrais contínuas. Ela serve para excluir processos com saltos em instantes fixos (não aleatórios). Descontinuidades podem ocorrer em tempos aleatórios.

\section{Medida de Salto e Medida de Lévy}

Para todo processo cádlág $\left\{X_{t}\right\}_{t \geq 0}$ com valores em $\mathbb{R}^{d}$ faz sentido definirmos $\Delta X_{t}=X_{t}-X_{t-\text {. }}$

A medida de contagem

$$
J_{X}(B)=\#\left\{\Delta X_{t} \neq 0,\left(t, \Delta X_{t}\right) \in B\right\}
$$

para $B \in \mathcal{B}\left([0, \infty) \times \mathbb{R}^{d}\right)$, é denominada medida de salto de $X$. Aqui, $\mathcal{B}$ denota os Borelianos.

Seja $\left\{X_{t}\right\}_{t \geq 0}$ um processo de Lévy em $\mathbb{R}^{d}$. Definimos a medida $\nu$ em $\mathbb{R}^{d}$, para $A \in \mathcal{B}\left(\mathbb{R}^{d}\right)$ por

$$
\nu(A)=\mathbb{E}\left[\#\left\{t \in[0,1]: \Delta X_{t} \neq 0, \Delta X_{t} \in A\right\}\right]=\mathbb{E}\left[J_{X}([0,1] \times A)\right]
$$

como sendo a medida de Lévy de $X$. Esta medida pode ser interpretada como a quantidade média do número de saltos com dimensões em $A$, em uma unidade de tempo.

\section{Decomposição de Lévy-Itô}

Para $\left\{X_{t}\right\}_{t \geq 0}$ um processo de Lévy em $\mathbb{R}^{d}$ com medida de Lévy $\nu$, a decomposição de Lévy-Itô nos dá que

- $\nu$ é uma medida de Radon em $\mathbb{R}^{d} \backslash\{0\}$ e satisfaz

$$
\int_{|x| \leq 1}|x|^{2} \nu(d x)<\infty \text { e } \int_{|x| \geq 1} \nu(d x)<\infty
$$

- $J_{X}$ é uma medida aleatória de Poisson em $[0, \infty) \times \mathbb{R}^{d}$ com intensidade $\nu(x) d t$.

- Existem $\gamma \in \mathbb{R}^{d}$ e um MB d-dimensional $\left\{B_{t}\right\}_{t \geq 0}$ tal que, 


$$
\begin{array}{r}
X_{t}=\gamma t+B_{t}+\int_{|x| \geq 1, s \leq t} x J_{X}(d s \times d x)+ \\
\lim _{\varepsilon \searrow 0} \int_{\varepsilon \leq|x|<1, s<t} x \tilde{J}_{X}(d s \times d x),
\end{array}
$$

onde $\tilde{J}_{X}$ é a medida de Poisson compensada pela sua média.

\section{Representação de Lévy-Khintchine}

Podemos falar da função característica do vetor aleatório $X_{t}$ que devido a decomposição de Lévy-Itô resulta em

$$
\mathbb{E}\left[\exp \left(i<z, X_{t}>\right)\right]=\exp (t \psi(z)), \quad z \in \mathbb{R}^{d}
$$

com

$$
\begin{aligned}
\psi(z) & =-\frac{1}{2}\langle z, \boldsymbol{\Sigma} z>+i<\gamma, z> \\
& +\int_{\mathbb{R}^{d}}\left(\exp (i<z, x>)-1-i<z, x>\mathbf{1}_{\{|x| \leq 1\}}\right) \nu(d x)
\end{aligned}
$$

onde $\Sigma$ é a matriz de covariâncias do MB que compõe o processo de Lévy.

Com isso temos que o processo de Lévy pode ser parametrizado pela tripla $(\Sigma, \nu, \gamma)$ resultante da fórmula de Lévy-Khintchine.

\section{Cópulas para Vetores Aleatórios}

A distribuição de um vetor aleatório d-dimensional $\{\mathbf{X}\}$ é dada pela sua função de densidade acumulada (c.d.f.)

$$
F(\mathbf{x})=\mathbb{P}[\mathbf{X} \leq \mathbf{x}]
$$

Por distribuição marginal ou marginal de $\left(X_{1}, \ldots, X_{d}\right)$ entendemos as distribuições de $X_{1}, \ldots$ e $X_{d}$ separadamente. Estas distribuições marginais são descritas pelas suas funções de distribuições $F_{X_{k}}\left(x_{k}\right)=\mathbb{P}\left[X_{k} \leq x_{k}\right], k=1, \ldots, d$ que são obtidas a partir da c.d.f. d-dimensional:

$$
F_{X_{k}}\left(x_{k}\right)=F\left(\infty, \ldots, \infty, x_{k}, \infty, \ldots, \infty\right)
$$

A distribuição de um vetor aleatório d-dimensional, pode ser "decomposta" em suas distribuições marginais e a estrutura de dependência entre essas distribuições marginais. Dois vetores com as mesmas distribuições marginais, mas com estruturas de dependência diferentes possuem distribuições distintas. Desta maneira, um vetor aleatório com distribuições marginais $F_{X_{1}}\left(x_{1}\right), \ldots$ e $F_{X_{d}}\left(x_{d}\right)$, 
será igual a $F(\mathbf{x})=\prod_{k=1}^{d} F_{X_{k}}\left(x_{k}\right)$ se os seus componentes forem independentes e, $F(\mathbf{x})=\min _{k=1, \ldots, d}\left\{F_{X_{k}}\left(x_{k}\right)\right\}$ se eles forem perfeitamente dependentes, i.e., um dos componentes é uma função determinística estritamente crescente dos outros componentes.

A cópula de um vetor aleatório d-dimensional é uma função d-variada que caracteriza a estrutura de dependência e não depende das marginais. A c.d.f. pode ser obtida por meio da cópula $C(\mathbf{x})$ e de suas marginais, i.e., $F(\mathbf{x})=$ $C\left(F_{X_{1}}\left(x_{1}\right), \ldots, F_{X_{d}}\left(x_{d}\right)\right)$.

Podemos mostrar que a cópula de uma distribuição é invariante a transformações estritamente crescentes dos componentes do vetor aleatório (vide Nelsen (1999)). Assim, para todo conjunto de $d$ funções estritamente crescentes $f_{1}, \ldots$ e $f_{d}$, a cópula de $\left(X_{1}, \ldots, X_{d}\right)$ é a mesma que a cópula de $\left(f_{1}\left(X_{1}\right), \ldots, f_{d}\left(X_{d}\right)\right)$. $\mathrm{O}$ termo estrutura de dependência refere-se as características de uma distribuição que não dependem das marginais e, ao combinar esta estrutura às marginais é possível reconstruir inteiramente a distribuição. Uma abordagem entrópica de medida de dependência é sugerida por Joe (1989).

Muito tem sido feito em termos de aplicações de cópulas ordinárias em Finanças. Apenas para citar alguns exemplos, temos Rodriguez (2004), Cherubini e Luciano (2002) e Cherubini et alii (2004). Mencionamos também Rosenberg (1999) por uma razão histórica. Ele foi um dos primeiros trabalhos a estudar o apreçamento de papéis multidimensionais usando cópulas.

Vamos agora formalizar a c.d.f.. A primeira propriedade está relacionada com a idéia de volume de uma função crescente para funções de dimensões múltiplas:

Definição 1 F-Volume Seja $F$ uma função real $d$-variada com domínio $\mathcal{D}(F)$. Para $a, b \in \overline{\mathbb{R}}^{d}$, tal que $a \leq b$, i.e., $a_{k} \leq b_{k}$ para $k=1, \ldots, d$, seja $\left.\left.B=\right] a ; b\right]$ uma caixa $d$-dimensional, cujos vértices pertencem a $\mathcal{D}(F)$. O $F$-volume de $B$ é definido como

$$
V_{F}(B)=\sum \operatorname{sinal}(c) F(c)
$$

sendo que a soma é realizada em todos os $2^{d}$ vértices $c \in \overline{\mathbb{R}}^{d}$ de $B$ e, sinal $(c)$ é definido como

$$
\text { sinal }(c) \triangleq\left\{\begin{aligned}
1, & \text { se } c_{k}=a_{k} \text { em quantidade par } \\
-1, & \text { se } c_{k}=a_{k} \text { em quantidade ímpar }
\end{aligned}\right.
$$

\section{Definição 2 função d-crescente, função aderente}

1. Uma função real $F$ d-variada é denominada d-crescente se $V_{F}(B) \geq 0$ para todas as d-caixas $B$ cujos vértices pertencem à $\mathcal{D}(F)$.

2. Seja $\mathcal{D}(F)=S_{1} \times \cdots \times S_{d}$ o domínio da função $F$. Para cada $S_{k}$, seja $a_{k}=\inf S_{k}$, para $a_{k} \in \overline{\mathbb{R}} . F$ é uma função aderente se $F(t)=0, t \in \overline{\mathbb{R}}^{d}$, para todo $t \in \mathcal{D}(F)$ existir $t_{k}=a_{k}$ pelo menos para um k.Isto equivale a dizer que $F$ é aderente se ela se anular na borda inferior de seu domínio. 
Definição 3 Cópula Uma cópula $d$-dimensional é uma função $C$ com domínio $\mathcal{D}(C)=[0 ; 1]^{d}$, tal que:

1. C é uma função aderente e d-crescente.

2. $C$ possui marginais $C_{k}, k=1, \ldots, d$, que satisfaz $C_{k}(u)=u$ para todo $u \in[0 ; 1]$.

Cópulas podem ser interpretadas como funções de distribuições com marginais uniformes.

Teorema 4 Sklar Seja $F$ uma função de distribuição $d$-dimensional com marginais $F_{1}, \ldots, F_{d}$. Assim, existe uma cópula $d$-dimensional $C$, tal que, para todo $x \in \overline{\mathbb{R}}^{d}$,

$$
F\left(x_{1}, \cdots, x_{d}\right)=C\left(F_{1}\left(x_{1}\right), \cdots, F_{n}\left(x_{d}\right)\right)
$$

Se $F_{1}, \ldots, F_{d}$ são todas contínuas, então $C$ é única, caso contrário, $C$ será unicamente determinada em $\mathcal{D}(C)=\mathcal{I}\left(F_{1}\right) \times \ldots \times \mathcal{I}\left(F_{d}\right)$. De maneira inversa, se $C$ é uma $d$-cópula e $F_{1}, \ldots, F_{d}$ são funções de distribuições, então, a função $F$ definida pela equação (2) é um função de distribuição $d$-dimensional com marginais $F_{1}, \ldots, F_{d}$.

Conforme Kolev et alii (2005), uma consequiência da Definição 3, é possibilidade de escrever a Cópula $d$-dimensional da seguinte maneira:

$$
C\left(F_{1}\left(x_{1}\right), \cdots, F_{n}\left(x_{d}\right)\right)=\prod_{i=1}^{d} F_{i}\left(x_{i}\right)+D\left(F_{1}\left(x_{1}\right), \cdots, F_{d}\left(x_{d}\right)\right)
$$

para $D\left(F_{1}\left(x_{1}\right), \cdots, F_{d}\left(x_{n}\right)\right) \in[0 ; 1]^{d}$ e $D\left(F_{1}\left(x_{1}\right), \cdots, F_{d}\left(x_{d}\right)\right)$ representa toda a informação da dependência de $\left\{X_{1}, \cdots, X_{d}\right\}$.

Uma classe de cópulas ordinárias bastante usada em Finanças, é a Arquimedeana, que pode ser definida pela seguinte proposição:

Proposição 5 Seja $\phi:[0 ; 1] \longrightarrow[0 ; \infty]$ uma função contínua estritamente crescente, então,

$$
C(u, v)=\phi^{-1}(\phi(u)+\phi(v))
$$

é uma cópula Arquimediana se e somente se $\phi$ for convexa e $\phi(0) \triangleq 1$. $\phi$ é conhecida como a função geradora da família.

Uma prova desta proposição pode ser encontrada em Nelsen (1999, p.90). Para a família Clayton, $\phi(t)=\left(t^{-\phi}-1\right) / \phi$ e para a cópula independente $C_{\perp}$, $\phi(t)=-\ln (t)$. Para conhecer outras famílias de cópulas pertencentes a classe Arquimedeanas consultar Nelsen (1999, pp.94-97). 


\section{Dependência para Processos de Lévy}

Vamos agora discutir a modelagem multivariada concebida usando o Processo de Lévy. Modelamos a estrutura de dependência separadamente das marginais e para empreender a primeira parte usamos a cópula. Por uma questão didática vamos inicialmente desenvolver o caso bidimensional e depois damos a versão multidimensional.

Para cada $t>0$ fixado, temos que o processo de Lévy bidimensional $\left(X_{t}, Y_{t}\right)$ é um vetor aleatório. Assim, a estrutura de dependência deste vetor pode ser parametrizada pela cópula $C_{t}$ associada a $\left(X_{t}, Y_{t}\right)$ para cada $t>0$. Entretanto, esta abordagem apresenta desvantagens:

1. Salvo no caso dos processos estáveis, a cópula $C_{t}$ pode depender de $t$. $C_{s}$ para certo $s \neq t$ não pode, em geral, ser calculada a partir de $C_{t}$, pois ela também depende de suas marginais.

2. Para uma dada distribuição infinitamente divisível de $X_{t}$ e $Y_{t}$, não é possível definir em quais circunstâncias a cópula $C_{t}$ representará uma distribuição bidimensional infinitamente divisível.

3. As distribuições dos componentes de um processo de Lévy multidimensional são usualmente especificadas pelas suas medidas de Lévy. Assim, seria estranho usar cópulas de distribuições de probabilidade.

A propriedade da cópula de variáveis aleatórias serem invariantes às transformações estritamente crescentes das marginais, não é adequada para caracterizar o conceito de dependência no contexto dos processos de Lévy. A propriedade de divisibilidade infinita de uma variável aleatória é destruída por transformações estritamentes crescentes sobre si mesma. Assim, é necessário redefinir o conceito de dependência, estabelecendo alguma outra forma de transfomação, que mantenha as propriedades da estrutura dinâmica de um processo de Lévy.

Uma maneira de driblar essas dificuldades é recorrer a representação de LévyKhintchine de forma a descrever a dependência em termos da tripla $(\gamma, \boldsymbol{\Sigma}, \nu)$ do processo.

Claro que usando esta representação é possível caracterizar a dependência via subordinação de um MB por um processo de Lévy crescente conforme mencionado na introdução. Aqui, o interesse é fazer a caracterização via cópulas.

Sabemos pela Decomposição de Lévy-Itô que o termo contínuo do processo de Lévy é independente do termo que produz os saltos. Com isso, é possível modelar a dependência dos saltos de maneira separada da parte contínua. Como a estrutura de dependência da parte contínua de um processo de Lévy (MB) é inteiramente caracterizada pela matriz de covariâncias e como este tipo de dependência é muito bem conhecida, a partir deste ponto vamos considerar apenas os processos de Lévy sem o componente gaussiano, i.e., com $\Sigma=0$. Em outras palavras, usaremos cópulas para caracterizar dependências dos saltos nas trajetórias do processo. 
Para dar uma noção de como a dependência pode ser caracterizada pela tripla $(\gamma, \boldsymbol{\Sigma}, \nu)$ do processo mostramos em seguida como suas marginais e a independência entre elas podem ser representadas em termos de sua medida de Lévy.

Proposição 6 Marginais do Processo de Lévy Seja $\left(X_{t}, Y_{t}\right)_{t>0}$ um processo de Lévy com tripé $(\gamma, \Sigma, \nu)$. A marginal $\left\{X_{t}\right\}_{t \geq 0}$ possui tripla caracterizadora $\left(\gamma_{X}, \Sigma_{X}, \nu_{X}\right)$, dada por:

$$
\begin{aligned}
\Sigma_{X} & =\Sigma_{11} \\
\nu_{X}(B) & =\nu(B \times]-\infty ; \infty[), \forall B \in \mathcal{B}(\mathbb{R}) \\
\gamma_{X} & =\gamma_{1}+\int_{\mathbb{R}^{2}} x\left(\mathbf{1}_{\left\{x^{2} \leq 1\right\}}-\mathbf{1}_{\left\{x^{2}+y^{2} \leq 1\right\}}\right) \nu(d x \times d y)
\end{aligned}
$$

Isto significa que as marginais de uma medida de Lévy podem ser calculadas da mesma maneira que as marginais de uma medida de probabilidade.

O resultado segue diretamente das propriedades de uma transformação linear de um processo de Lévy como vistas em Cont e Tankov (2004), Teorema 4.1, p.105.

\section{Proposição 7 Independência dos Processos de Lévy}

Seja $\left(X_{t}, Y_{t}\right)_{t>0}$ um processo de Lévy com medida de Lévy $\nu$ sem o termo gaussiano. Seus componentes são independentes se e somente se o suporte dev está contido no conjunto $\{\{x, y\}: x y=0\}$, i.e., se e somente se os componentes não apresentam saltos no mesmo instante de tempo. Neste caso,

$$
\nu(B)=\nu_{X}\left(B_{X}\right)+\nu_{Y}\left(B_{Y}\right)
$$

sendo que $B_{X}=\{x:\{x, 0\} \in B\}$ e $B_{Y}=\{y:\{0, y\} \in B\}$, e $\nu_{X}$ e $\nu_{Y}$ são as medidas de Lévy de $\left\{X_{t}\right\}_{t \geq 0}$ e $\left\{Y_{t}\right\}_{t \geq 0}$.

Para uma prova do resultado veja Cont e Tankov (2004), Proposição 5.3, p.144.

\subsection{Cópulas para Processos de Lévy de Saltos Positivos}

Conforme Cont e Tankov (2004, p.145), para parametrizar a dependência entre os saltos de processos de Lévy, a medida de Lévy executará o mesmo papel que a medida de probabilidade executa para as variáveis aleatórias. Assim, para modelar a dependência, é necessário criar cópulas para as medidas de Lévy. A principal diferença da cópula ordinária para a cópula de uma medida de Lévy é que esta última não é necessariamente finita, i.e., ela tem uma singularidade no zero que não é integrável. Também, cópulas de Lévy são definidas em intervalos infinitos, ao invés de intervalos em $[0 ; 1]^{2}$. Cópulas bidimensionais de Lévy para processos 
com saltos positivos são funções $C^{\mathcal{L}}:[0 ; \infty]^{2} \longrightarrow[0 ; \infty]$ e, de uma forma geral, elas são funções $C^{\mathcal{L}}: \overline{\mathbb{R}}^{2} \longrightarrow \overline{\mathbb{R}}$.

Para o caso de processos de Lévy com saltos positivos, o papel da função de distribuição é exercido pela integral caudal.

\subsection{Integral caudal e medidas de Lévy}

Para toda medida de Lévy $\nu$ em $[0 ; \infty[$, definimos a sua integral caudal da seguinte maneira:

$$
\begin{aligned}
U\left(x_{1}, x_{2}\right) & =0 \text { se } x_{1}=\infty \text { ou } x_{2}=\infty \\
U\left(x_{1}, x_{2}\right) & =\nu\left(\left[x_{1} ; \infty\left[,\left[x_{2} ; \infty[) \text { para }\left\{x_{1}, x_{2}\right\} \in \mathbb{R}_{+}^{2} \backslash\{0\}\right.\right.\right.\right. \\
U(0,0) & =\infty
\end{aligned}
$$

Uma integral caudal bidimensional, com as propriedades de continuidade apresentadas na Definição 2, define uma medida positiva $\nu$ em $\mathbb{R}_{+}^{2} \backslash\{0\}$. Entretanto, para que seja uma medida de Lévy, $\nu$ deve satisfazer a condição de integrabilidade apresentada na Decomposição de Lévy-Itô:

$$
\int_{[0 ; \infty]^{2}}\left(|x|^{2} \wedge 1\right) \nu(d x)<\infty \Longleftrightarrow \int_{[0 ; \infty]^{2}}\left(|x|^{2} \wedge 1\right) U(d x)<\infty
$$

sendo que $U$ é uma medida de Lebesgue, que existe porque $U$ é uma função crescente. O seguinte lema mostra quando esta condição de integrabilidade é satisfeita.

Lema 8 Seja $U$ uma integral caudal bidimensional com marginais $U_{1}$ e $U_{2}$. $U$ define uma medida de Lévy em $\mathbb{R}_{+}^{2} \backslash\{0\}$, i.e., a condição de integrabilidade apresentada na equação (6) é satisfeita se e somente se as marginais de $U$ correspondem a medidas de Lévy em $\mathbb{R}_{+}$, i.e., para $k \in\{1,2\}$,

$$
\int_{0}^{1} x^{2} U_{k}(d x)<\infty
$$

Aos interessados na prova referenciamos Cont e Tankov (2004), Lema 5.2, p.147.

Teorema 9 Seja $\left(X_{t}, Y_{t}\right)_{t>0}$ um processo de Lévy bidimensional com saltos positivos, integral caudal $U$ e marginais da integral caudal $U_{1}$ e $U_{2}$. Existe uma cópula de Lévy bidimensional $C^{\mathcal{L}}$ que caracteriza a estrutura de dependência de $\left(X_{t}, Y_{t}\right)_{t \geq 0}$, i.e., para todo $x_{1}, x_{2} \in[0 ; \infty]$,

$$
U\left(x_{1}, x_{2}\right)=C^{\mathcal{L}}\left(U_{1}\left(x_{1}\right), U_{2}\left(x_{2}\right)\right)
$$

Se $U_{1}$ e $U_{2}$ forem contínuas, esta cópula de Lévy é única. Caso contrário, ela será unicamente determinada em $\mathcal{D}\left(C^{\mathcal{L}}\right)=\mathcal{I}\left(U_{1}\right) \times \mathcal{I}\left(U_{2}\right)$. No sentido inverso, 
seja $\left\{X_{t}\right\}_{t \geq 0} e\left\{Y_{t}\right\}_{t \geq 0}$ dois processos de Lévy unidimensionais com saltos positivos que possuem integrais caudais $U_{1}$ e $U_{2}$ e, $C^{\mathcal{L}}$ é uma cópula positiva de Lévy bidimensional. Assim, existe um processo de Lévy com cópula de Lévy $C^{\mathcal{L}}$ com integrais caudais marginais $U_{1}$ e $U_{2}$, sendo que a integral caudal será dada pela equação (8).

A prova deste teorema pode ser encontrada em Cont e Tankov (2004). A primeira parte deste teorema dispõe que todos os tipos de dependência existentes entre processos de Lévy, incluindo dependência completa e independência, podem ser representadas por cópulas de Lévy e, a segunda parte do teorema, mostra que é possível construir modelos baseados em processos de Lévy multivariados ao especificar a estrutura de dependência dos saltos separadamente das distribuições unidimensionais dos componentes do modelo. A distribuição dos componentes podem apresentar estruturas bastante diferentes e, em particular, é possível acoplar processos compostos de Poisson com os de atividades infinitas.

Conforme Cont e Tankov (2004, p.148), quando a dependência é especificada por meio de uma cópula de Lévy e, a cópula e as integrais caudais marginais são suficientemente suaves, a medida de Lévy pode ser calculada pela seguinte diferenciação:

$$
\nu\left(x_{1}, x_{2}\right)=\left.\frac{\partial^{2} C^{\mathcal{L}}\left(y_{1}, y_{2}\right)}{\partial y_{1} \partial y_{2}}\right|_{y_{1}=U_{1}\left(x_{1}\right), y_{1}=U_{2}\left(x_{2}\right)} \nu_{1}\left(x_{1}\right) \nu_{2}\left(x_{2}\right)
$$

\subsection{Exemplos de cópulas de Lévy positivas}

Apresentamos em seguida as cópulas de Lévy correspondentes às várias estruturas de dependência básicas.

Exemplo 10 Independência Seja $\left(X_{t}, Y_{t}\right)_{t>0}$ um processo de Lévy com componentes independentes. Pela Proposição 7, a medida de Lévy deste processo é dada por

$$
\begin{aligned}
\nu(B) & =\nu_{1}\left(B_{X}\right)+\nu_{2}\left(B_{Y}\right) \\
\nu\left(\left[x_{1} ; \infty\right] \times\left[x_{2} ; \infty\right]\right) & =\nu_{1}\left(\left[x_{1} ; \infty\right]\right)+\nu_{2}\left(\left[x_{2} ; \infty\right]\right) \\
& =\nu\left(\left[x_{1} ; \infty\right] \times[0 ; \infty]\right) \mathbf{1}_{\left\{x_{2}=0\right\}} \\
& +\nu\left([0 ; \infty] \times\left[x_{2} ; \infty\right]\right) \mathbf{1}_{\left\{x_{1}=0\right\}} \\
U\left(x_{1}, x_{2}\right) & =U\left(x_{1}, 0\right) \mathbf{1}_{\left\{x_{2}=0\right\}}+U\left(0, x_{2}\right) \mathbf{1}_{\left\{x_{1}=0\right\}} \\
& =U_{1}\left(x_{1}\right) \mathbf{1}_{\left\{x_{2}=0\right\}}+U_{2}\left(x_{2}\right) 1_{\left\{x_{1}=0\right\}}
\end{aligned}
$$

A cópula de Lévy de processos independentes é calculada usando a equação (8): 


$$
\begin{aligned}
C_{\perp}^{\mathcal{L}}\left(y_{1}, y_{2}\right) & =U\left(U_{1}^{-1}\left(y_{1}\right), U_{2}^{-1}\left(y_{2}\right)\right) \\
& =U_{1}\left(U_{1}^{-1}\left(y_{1}\right)\right) \mathbf{1}_{\left\{U_{2}^{-1}\left(y_{2}\right)=0\right\}} \\
& +U_{2}\left(U_{2}^{-1}\left(y_{2}\right)\right) \mathbf{1}_{\left\{U_{1}^{-1}\left(y_{1}\right)=0\right\}} \\
& =y_{1} \mathbf{1}_{\left\{y_{2}=\infty\right\}}+y_{2} \mathbf{1}_{\{y 1=\infty\}}
\end{aligned}
$$

Quando $U_{1}$ e $U_{2}$ não forem contínuas, a equação (9) será apenas uma das possibilidades de cópulas de Lévy para o processo bidimensional $\left(X_{t}, Y_{t}\right)_{t \geq 0}$.

No outro extremo temos a dependência completa dos saltos ou comonotonicidade dos componentes de um processo de Lévy com saltos positivos. Para discutila vamos definir o conceito de um conjunto crescente.

Definição 11 Um subconjunto $S \subset \overline{\mathbb{R}}^{2}$ é denominado crescente se para todo dois vetores $\left\{v_{1}, v_{2}\right\} \in S$ e $\left\{u_{1}, u_{2}\right\} \in S$, ou $v_{k}<u_{k}$ ou $v_{k}>u_{k}$ para $\forall k$.

Um elemento de um conjunto crescente é completamente determinado por apenas uma coordenada, o que motiva a seguinte definição de dependência dos saltos:

Definição 12 Seja $X=\left(X_{t}^{1}, X_{t}^{2}\right)_{t>0}$ um processo de Lévy com saltos positivos. Seus saltos são perfeitamente dependentes ou comonotônicos se existir um subconjunto crescente $S \in] 0 ; \infty\left[{ }^{2}\right.$, de modo que, todo salto $\Delta X$ é um elemento de $S$.

Se os saltos de dois processos de Lévy que se movem puramente por saltos forem perfeitamente dependentes, a trajetória de um deles pode ser reconstruída por meio do conhecimento da trajetória do outro.

Proposição 13 (Dependência Perfeita) Seja $X=\left(X_{t}^{1}, X_{t}^{2}\right)_{t \geq 0}$ um processo de Lévy com saltos positivos. Se os seus saltos forem completamente dependentes, então, a (possível) cópula de Lévy de $X$ é uma cópula de Lévy completamente dependente, que é definida por:

$$
C_{\|}^{\mathcal{L}}\left(x_{1}, x_{2}\right)=\min \left(x_{1}, x_{2}\right)
$$

De maneira inversa, se uma cópula de Lévy de $X$ é dado por $C_{\|}^{\mathcal{L}}$ e, as integrais caudais de seus componentes forem contínuas, então, os saltos de $X$ são completamente dependentes.

Demonstração Os saltos de $X$ são perfeitamente dependentes se e somente se existir um subconjunto crescente $S \in] 0 ; \infty\left[^{2}\right.$, tal que, a medida de Lévy $\nu$ está concentrada em $S$. Desta maneira, para todo $x=\left(x_{1}, x_{2}\right)$ e $y=\left(y_{1}, y_{2}\right)$,

$$
U(x)=\nu(\{y \geq x\} \cap S)=\int_{\{y \geq x\} \cap S} \nu(d y)
$$


Por meio desta igualdade é possível obter as desigualdades para as marginais:

$$
U_{k}\left(x_{k}\right)=\nu\left(\left\{y_{k} \geq x_{k}\right\} \cap S\right)=\int_{\left\{y_{k} \geq x_{k}\right\} \cap S} \nu(d y), k \in\{1,2\}
$$

Conforme Kallsen e Tankov (2006) é possível verificar que $U(x) \leq U_{k}\left(x_{k}\right)$, para qualquer $k \mathrm{e}$,

$$
\left\{y \in S: y_{k} \geq x_{k}\right\} \cap S=\{y \in S: y \geq x\} \cap S
$$

para algum $k$, o que implica em

$$
U(x)=\min \left(U_{1}\left(x_{1}\right), U_{2}\left(x_{2}\right)\right)
$$

Por outro lado, quando a integral caudal de $X$ apresenta a forma da equação (10) a medida de Lévy está concentrada no conjunto $\left\{\left(x_{1}, x_{2}\right)\right.$ : $\left.U_{1}\left(x_{1}\right)=U_{2}\left(x_{2}\right)\right\}$. Se as integrais caudais $U_{1}$ e $U_{2}$ forem contínuas, então este conjunto é crescente.

Exemplo 14 Dependência de Processos Estáveis Cópulas de Lévy de processos estáveis satisfazem

$$
C^{\mathcal{L}}\left(c x_{1}, c x_{2}\right)=c C^{\mathcal{L}}\left(x_{1}, x_{2}\right), \forall c>0
$$

ou seja, elas são funções homogêneas de ordem 1. Portanto, pela Decomposição de Lévy-Itô, para a medida de Lévy de processos estáveis vale

$$
\nu(B)=c^{\alpha} \nu(c B), \forall c>0, \forall B \in \mathcal{B}\left(\mathbb{R}^{2}\right)
$$

de modo que a integral caudal satisfaz

$$
U\left(c x_{1}, c x_{2}\right)=c^{-\alpha} U\left(x_{1}, x_{2}\right)
$$

Concluimos que a cópula de Lévy de um processo estável é homogênea de ordem 1, ao substituir as marginais $\alpha$-estáveis nesta última fórmula. Antecipamos que este exemplo é uma cópula de Lévy da família Arquimediana com gerador $\phi(x)=\frac{\exp \{-x\}}{1-\exp \{-x\}}$ (Proposição 16).

\subsection{Construção de cópulas de Lévy positivas}

A fórmula

$$
C^{\mathcal{L}}\left(y_{1}, y_{2}\right)=U\left(U_{1}^{-1}\left(y_{1}\right), U_{2}^{-1}\left(y_{2}\right)\right)
$$

não coopera na interpretação das cópulas de Lévy, pois ela não é construtiva. Isto motivou desenvolver outras maneiras de obtê-las: 
Proposição 15 Seja $C$ uma 2-cópula (ordinária) e $f(x)$ uma função convexa crescente definida como $f:[0 ; 1] \longrightarrow[0 ; \infty]$.

$$
C^{\mathcal{L}}(x, y)=f\left(C\left(f^{-1}(x), f^{-1}(y)\right)\right)
$$

define uma cópula de Lévy positiva bidimensional.

Demonstração $C^{\mathcal{L}}$ ser uma função 2 -crescente provém da Definição $3 . C^{\mathcal{L}}$ ser aderente e ter as propriedades das marginais são de constatação imediata.

Um exemplo de função $f:[0 ; 1] \longrightarrow[0 ; \infty]$ que pode ser utilizada nesta proposição é dada por $f(x)=\frac{x}{1-x}$. Por analogia, cópulas Arquimedeanas permitem construir cópulas de Lévy Arquimedeanas.

Proposição 16 Seja $\phi(u)$ uma função convexa estritamente descrescente $\phi$ : $[0 ; \infty] \longrightarrow[0 ; \infty]$, tal que, $\phi(0)=\infty e \phi(\infty)=0$. Então,

$$
C^{\mathcal{L}}(x, y)=\phi^{-1}(\phi(x)+\phi(y))
$$

fornece-nos uma cópula de Lévy positiva bidimensional.

Demonstração Segue novamente da Definição 3, dado que $\phi^{-1}(-u)$ é crescente e convexa.

Exemplo 17 Família Clayton de cópulas de Lévy Para $\phi(u)=u^{-\theta} \operatorname{com} \theta>0$, obtém-se a seguinte família paramétrica de cópulas de Lévy:

$$
C_{\theta}^{\mathcal{L}}(u, v)=\left(u^{-\theta}+v^{-\theta}\right)^{-1 / \theta}
$$

que relembra a família de cópulas de Clayton (veja Nelsen $(1999$, p.96)). Como casos limites temos a completa dependência $(\theta \longrightarrow \infty)$ e a independência $(\theta \downarrow 0)$.

Exemplo 18 Família Frank de cópulas de Lévy Para $\phi(u)=-\log (1-$ $\exp (-\theta u)) \operatorname{com} \theta>1$, tem-se a seguinte família de cópulas de Lévy:

$$
C_{\theta}^{\mathcal{L}}(u, v)=\frac{-1}{\theta} \log \left\{1-\left(e^{-\theta u}-1\right)\left(e^{-\theta v}-1\right)\right\}
$$

que relembra a família de cópulas de Frank (veja Nelsen (1999, p.97)). Nos casos limites temos a completa dependência $(\theta \longrightarrow \infty)$ e a independência $(\theta \downarrow 1)$.

Até onde sabemos esta proposta de extensão da família Frank de cópulas é inédita. 


\subsection{Interpretação probabilística}

As cópulas de Lévy não são funções de distribuições, mas as suas derivadas possuem uma interpretação probabilística. Kallsen e Tankov (2006), provam o seguinte lema:

Lema 19 Seja $C^{\mathcal{L}}$ uma cópula de Lévy bidimensional. Para todo $x \in[0 ; \infty]$, a função

$$
C_{x}^{\mathcal{L}}(y)=\frac{\partial}{\partial x} C^{\mathcal{L}}(x, y)
$$

existe e é contínua para todo $y \in[0 ; \infty]$ a menos de um conjunto enumerável. Desta maneira, $C_{x}^{\mathcal{L}}(y)$ é uma função de distribuição de uma variável aleatória positiva, de maneira que, $C_{x}^{\mathcal{L}}(y)$ é uma função crescente e satisfaz $C_{x}^{\mathcal{L}}(0)=0 e$ $C_{x}^{\mathcal{L}}(\infty)=1$.

A interpretação desse resultado é objeto do teorema seguinte, que mostra que $C^{\mathcal{L}}$ determina uma distribuição de saltos (transformados) do segundo componente, condicionado ao tamanho do salto do primeiro componente. Esta propriedade será útil para simular processos de Lévy. Para uma prova veja Taleb (1997).

Teorema 20 Seja $\left(X_{t}, Y_{t}\right)_{t>0}$ um processo de Lévy bidimensional com saltos positivos, possuindo integrais caudais marginais $U_{1}$ e $U_{2}$ e cópula de Lévy igual a $C^{\mathcal{L}}$. Seja $\Delta X_{t}$ e $\Delta Y_{t}$ o tamanho dos saltos dos dois componentes do processo no instante de tempo $t$. Se $U_{1}$ possuir uma densidade não-negativa em $x, C_{U_{1}(x)}^{\mathcal{L}}$ é a função de distribuição de $U_{2}\left(\Delta Y_{t}\right)$ condicionada em $\Delta X_{t}=x$ :

$$
C_{U_{1}(x)}^{\mathcal{L}}(y)=\mathbb{P}\left[U_{2}\left(\Delta Y_{t}\right) \mid \Delta X_{t}=x\right]
$$

A extensão para o caso multivariado do que foi feito nesta seção é apresentada no apêndice.

\section{Cópulas de Processos de Lévy Gerais}

Vamos estender os conceitos para os processos de Lévy que apresentam saltos tanto positivos como negativos, como segue:

Definição $21 C^{\mathcal{L}}(x, y):[-\infty ; \infty]^{2} \longrightarrow[-\infty ; \infty]$ é uma cópula de Lévy se satisfizer as seguintes propriedades:

1. $C^{\mathcal{L}}$ é 2-crescente;

2. $C^{\mathcal{L}}(0, x)=C^{\mathcal{L}}(x, 0)=0, \forall x$;

3. $C^{\mathcal{L}}(x, \infty)-C^{\mathcal{L}}(x,-\infty)=C^{\mathcal{L}}(\infty, x)-C^{\mathcal{L}}(-\infty, x)=x$. 
Com isto observamos que a cópula de Lévy positiva pode ser estendida para uma cópula de Lévy geral ao estabelecermos que $C^{\mathcal{L}}(x, y)=0$ se $x<0$ ou $y<0$.

\subsection{Medidas de Lévy com densidades}

Começamos com este caso porque aí a modelagem é simples. Considere, inicialmente, a idéia de uma integral caudal de uma medida de Lévy definida em $\mathbb{R}$.

Definição 22 Seja $\nu$ uma medida de Lévy em $\mathbb{R}$. A integral caudal de $\nu$ é uma função $U: \overline{\mathbb{R}} \backslash\{0\} \longrightarrow[0 ; \infty]$ definida como

$$
\begin{aligned}
U(x) & =\nu([x ; \infty[), x \in] 0 ; \infty[ \\
U(x) & =-\nu([-\infty ; x[), x \in]-\infty ; 0[ \\
U(\infty) & =U(-\infty)=0
\end{aligned}
$$

Os sinais foram estabelecidos de maneira tal que a integral caudal é uma função decrescente tanto para $x \in] 0 ; \infty[$, como para $x \in]-\infty ; 0[$.

A construção de densidades de Lévy bidimensionais por meio de cópulas de Lévy suficientemente suaves e densidades de Lévy unidimensionais sai do resultado seguinte.

Proposição 23 Seja $C^{\mathcal{L}}$ uma cópula de Lévy bidimensional, contínua em $\overline{\mathbb{R}}^{2}$, tal que $\frac{\partial^{2} C^{\mathcal{L}}(u, v)}{\partial u \partial v}$ existe em $\mathbb{R}^{2}$ e sejam $U_{1}$ e $U_{2}$ as integrais caudais unidimensionais com densidades $\nu_{1}$ e $\nu_{2}$. Assim,

$$
\left.\frac{\partial^{2} C^{\mathcal{L}}(u, v)}{\partial u \partial v}\right|_{u=U_{1}\left(x_{1}\right), v=U_{2}\left(x_{2}\right)} \nu_{1}\left(x_{1}\right) \nu_{2}\left(x_{2}\right)
$$

é a densidade de Lévy de uma medida de Lévy com densidades de Lévy marginais $\nu_{1}$ e $\nu_{2}$

\subsection{Cópulas de Lévy generalizadas}

No caso da cópula de Lévy e as integrais caudais marginais não forem suficientemente suaves, a integral caudal multidimensional deve substituir a densidade de Lévy. A fim de evitar a singularidade da medida de Lévy na origem vamos desmembrar o domínio dessa medida em regiões (quadrantes ou cantos) similares a considerada na medida positiva e trabalhá-las separadamente. No caso unidimensional são necessárias duas integrais caudais: $U^{+}$e $U^{-}$e no caso bidimensional, quatro: $U^{++}, U^{+-}, U^{-+}$e $U^{--}$.

Definição 24 Seja $\nu$ uma medida de Lévy em $\mathbb{R}$. Esta medida apresenta duas integrais caudais, $U^{+}:[0 ; \infty] \longrightarrow[0 ; \infty]$ para a parte positiva e, $U^{-}:[-\infty ; 0] \longrightarrow$ $[-\infty ; 0]$ para a parte negativa, definidas como: 


$$
\begin{aligned}
U^{+}(x) & =\nu([x ; \infty[), x \in] 0 ; \infty[, \\
U^{+}(0) & =\infty, U^{+}(\infty)=0, \\
U^{-}(x) & =\nu(]-\infty, x]), x \in]-\infty ; 0[, \\
U^{-}(-\infty) & =0, U^{-}(0)=-\infty
\end{aligned}
$$

Seja $\nu$ uma medida de Lévy em $\mathbb{R}^{2}$ com integrais caudais marginais $U_{1}^{+}, U_{1}^{-}$, $U_{2}^{+}$e $U_{2}^{-}$. Esta medida possui quatro integrais caudais, i.e., $U^{++}, U^{+-}, U^{-+}$ e $U^{--}$, sendo que, cada integral caudal é definida no seu respectivo quadrante, incluindo o eixo das coordenadas, como segue:

$$
\begin{aligned}
& U^{++}(x, y)=\nu([x ; \infty[\times[y ; \infty[), x, y \in] 0 ; \infty[ \\
& \left.U^{+-}(x, y)=-\nu([x ; \infty[\times]-\infty, y]), x \in\right] 0 ; \infty[, y \in]-\infty ; 0[ \\
& \left.U^{-+}(x, y)=-\nu(]-\infty, x\right] \times[y ; \infty[), x \in]-\infty ; 0[, y \in] 0 ; \infty[ \\
& \left.\left.\left.\left.\left.U^{--}(x, y)=\nu(]-\infty, x\right] \times\right]-\infty, y\right]\right), x, y \in\right]-\infty ; 0[
\end{aligned}
$$

Se $x$ ou $y$ for igual a $-\infty$ ou $+\infty$, a integral caudal correspondente é igual a zero. Se $x$ ou $y$ for igual a 0 , a integral caudal satisfaz as seguintes condições marginais:

$$
\begin{aligned}
& U^{++}(x, 0)-U^{+-}(x, 0)=U_{1}^{+}(x) \\
& U^{-+}(x, 0)-U^{--}(x, 0)=U_{1}^{-}(x) \\
& U^{++}(0, y)-U^{-+}(0, y)=U_{2}^{+}(y) \\
& U^{+-}(0, y)-U^{--}(0, y)=U_{2}^{-}(y)
\end{aligned}
$$

Quando uma medida de Lévy bidimensional precisa mensurar qualquer eixo das coordenadas sua integral caudal não é unicamente determinada pois não fica claro qual das integrais caudais deve conter a densidade nos eixos. Desta maneira, conjuntos diferentes para as integrais caudais podem representar a mesma medida de Lévy.

Teorema 25 Seja $\nu$ uma medida de Lévy em $\mathbb{R}^{2}$ com suas integrais caudais marginais $U_{1}^{+}(x), U_{1}^{-}(x), U_{2}^{+}(y)$ e $U_{2}^{-}(y)$. Existe uma cópula de Lévy $C^{\mathcal{L}}$ satisfazendo,

$$
\begin{aligned}
& \left.U^{++}(x, y)=C^{\mathcal{L}}\left(U_{1}^{+}(x), U_{2}^{+}(y)\right), x, y \in\right] 0 ; \infty[ \\
& \left.U^{+-}(x, y)=C^{\mathcal{L}}\left(U_{1}^{+}(x), U_{2}^{-}(y)\right), x \in\right] 0 ; \infty[, y \in]-\infty ; 0[ \\
& \left.U^{-+}(x, y)=C^{\mathcal{L}}\left(U_{1}^{-}(x), U_{2}^{+}(y)\right), x \in\right]-\infty ; 0[, y \in] 0 ; \infty[ \\
& \left.U^{--}(x, y)=C^{\mathcal{L}}\left(U_{1}^{-}(x), U_{2}^{-}(y)\right), x, y \in\right]-\infty ; 0[
\end{aligned}
$$


Se as integrais caudais marginais são absolutamente contínuas e, $\nu$ não mensura os eixos das coordenadas, a cópula de Lévy é única. De maneira inversa, se $C^{\mathcal{L}}$ é uma cópula de Lévy e $U_{1}^{+}(x), U_{1}^{-}(x), U_{2}^{+}(y)$ e $U_{2}^{-}(y)$ são integrais caudais unidimensionais de medidas de Lévy então as fórmulas acima definem as integrais caudais da medida de Lévy bidimensional.

Demonstração A cópula de Lévy pode ser construída em cada um dos quatro quadrantes ao utilizar o Teorema 9 e o Lema 8. Com esta construção, é possível obter uma função 2 -crescente em cada um dos quadrantes, mas não para todo $\mathbb{R}^{2}$. Apesar disto, o fato de cada uma das quatro partes serem contínuas nos seus respectivos domínios, incluindo os eixos das coordenadas e serem iguais à zero nos extremos das caudas garante que $C^{\mathcal{L}}$ é uma função 2 -crescente em $\overline{\mathbb{R}}^{2}$.

Exemplo 26 Processos de Lévy Independentes Dado que a medida de Lévy bidimensional de um processo de Lévy com componentes independentes tem suporte nos eixos das coordenadas, a correspondente cópula de Lévy não é única. Neste caso, a Proposição 7 garante que para todo $x$ e $y$, tal que, $x y \neq 0$, integral caudal correspondente é igual a zero. Assim, $C^{\mathcal{L}}(x, y)=0$ se $x$ e y forem finitos. Pelas fórmulas (19) vemos que cada cópula de Lévy que satisfaça esta propriedade é independente. Exemplos de cópulas de Lévy independentes são $x \mathbf{1}_{\{y=\infty\}}+y \mathbf{1}_{\{x=\infty\}} e-x \mathbf{1}_{\{y=-\infty\}}-y \mathbf{1}_{\{x=-\infty\}}$.

Exemplo 27 Dependência Perfeita Para processos de Lévy com saltos de sinais arbitrários, é possível distinguir dois tipos de dependência completa. No primeiro (dependência completa positiva) existe um subconjunto crescente $S \subset \mathbb{R}^{2}$, tal que, cada salto $\Delta X$ de um processo bidimensional pertence ao conjunto $S$. Nesta situação, ao utilizar o mesmo método da Proposição 13, tem-se

$$
\begin{aligned}
& U^{++}(x, y)=\min \left(U_{1}^{+}(x), U_{2}^{+}(y)\right) \\
& U^{--}(x, y)=\min \left(-U_{1}^{-}(x),-U_{2}^{-}(y)\right) \\
& U^{+-}(x, y)=U^{-+}(x, y) \triangleq 0
\end{aligned}
$$

Desta maneira, $C_{\uparrow \uparrow}^{\mathcal{L}}(x, y)=\min (|x|,|y|) 1_{\{x y \geq 0\}}$ é uma possível cópula para este processo. No caso de dependência completa negativa, os saltos de um processo bidimensional devem estar contidos em um subconjunto descrescente em $\mathbb{R}^{2}$, i.e., o conjunto $S$, tal que para todo dois vetores $\left(v_{1}, v_{2}\right) \in S$ e $\left(u_{1}, u_{2}\right) \in S$, tanto $\nu_{1}>u_{1}$ e $v_{2}<u_{2}$ ou, $\nu_{1}<u_{1}$ e $v_{2}>u_{2}$. Neste caso:

$$
\begin{aligned}
& U^{+-}(x, y)=-\min \left(U_{1}^{+}(x),-U_{2}^{-}(y)\right) \\
& U^{-+}(x, y)=-\min \left(-U_{1}^{-}(x), U_{2}^{+}(y)\right) \\
& U^{++}(x, y)=U^{--}(x, y) \triangleq 0
\end{aligned}
$$


e $C_{\uparrow \downarrow}^{\mathcal{L}}(x, y)=-\min (|x|,|y|) 1_{\{x y \leq 0\}}$.

Exemplo 28 Familia Clayton Generalizada Considere a cópula de Lévy apresentada na equação (14). É possivel definir uma família de cópulas de Lévy uniparamétrica, que inclui a independência e as dependências completas positiva e negativa.

$$
C_{\theta}^{\mathcal{L}}(u, v)=\left\{\begin{array}{cr}
\left(|u|^{-\theta}+|v|^{-\theta}\right)^{-1 / \theta} \mathbf{1}_{\{u v \geq 0\}} & \text { se } \theta>0 \\
-\left(|u|^{\theta}+|v|^{\theta}\right)^{1 / \theta} \mathbf{1}_{\{u v \leq 0\}} & \text { se } \theta<0
\end{array}\right.
$$

Ao calcular os limites, tem-se que

$$
\begin{aligned}
& C_{\theta}^{\mathcal{L}} \longrightarrow C_{\uparrow \downarrow}^{\mathcal{L}} \text {, quando } \theta \longrightarrow-\infty \\
& C_{\theta}^{\mathcal{L}} \longrightarrow C_{\perp}^{\mathcal{L}}, \text { quando } \theta \longrightarrow 0 \\
& C_{\theta}^{\mathcal{L}} \longrightarrow C_{\uparrow \uparrow}^{\mathcal{L}}, \text { quando } \theta \longrightarrow \infty \mathbf{\square}
\end{aligned}
$$

Exemplo 29 Familia Frank Generalizada Considere agora a cópula de Lévy apresentada na equação (15). Neste caso definimos uma família de cópulas de Lévy uni-paramétrica, que também inclui a independência e as dependências completas positiva e negativa.

$$
C_{\theta}^{\mathcal{L}}(u, v)=\left\{\begin{array}{cc}
\frac{-1}{\theta} \log \left\{1-\left(e^{-\theta|u|}-1\right)\left(e^{-\theta|v|}-1\right)\right\} \mathbf{1}_{\{u v \geq 0\}} & \text { se } \theta>0 \\
\frac{1}{\theta} \log \left\{1-\left(e^{\theta|u|}-1\right)\left(e^{\theta|v|}-1\right)\right\} \mathbf{1}_{\{u v \leq 0\}} & \text { se } \theta<0
\end{array}\right.
$$

Tem-se, ao calcular os limites, que

$$
\begin{aligned}
& C_{\theta}^{\mathcal{L}} \longrightarrow C_{\uparrow \downarrow}^{\mathcal{L}} \text {, quando } \theta \longrightarrow-\infty \\
& C_{\theta}^{\mathcal{L}} \longrightarrow C_{\perp}^{\mathcal{L}} \text {, quando }|\theta|<\infty \\
& C_{\theta}^{\mathcal{L}} \longrightarrow C_{\uparrow \uparrow}^{\mathcal{A}} \text {, quando } \theta \longrightarrow \infty
\end{aligned}
$$

A dificuldade nessa construção reside no fato das Proposições 15 e 16 não poderem ser estendidas diretamente. Uma possível solução é agrupar cópulas de Lévy positivas, adaptadas de forma a especificar as dependências dos saltos de diferentes sinais separadamente. Ou seja, tomamos $C^{\mathcal{L}++}, C^{\mathcal{L}--}, C^{\mathcal{L}-+}$ e $C^{\mathcal{L}+-}$ cópulas de Lévy positivas e as reunimos segundo a expressão:

$$
\begin{aligned}
C^{\mathcal{L}}(x, y) & =C^{\mathcal{L}++}\left(c_{1}|x|, c_{2}|y|\right) \mathbf{1}_{\{x \geq 0, y \geq 0\}} \\
& +C^{\mathcal{L}--}\left(c_{3}|x|, c_{4}|y|\right) \mathbf{1}_{\{x \leq 0, y \leq 0\}} \\
& -C^{\mathcal{L}+-}\left(c_{5}|x|, c_{6}|y|\right) \mathbf{1}_{\{x \geq 0, y \leq 0\}} \\
& -C^{\mathcal{L}-+}\left(c_{7}|x|, c_{8}|y|\right) \mathbf{1}_{\{x \leq 0, y \geq 0\}}
\end{aligned}
$$


Note que $C^{\mathcal{L}}$ é 2 -crescente se as constantes $c_{1}, \ldots, c_{8}$ forem positivas. As condições marginais implicam em quatro restrições adicionais, e.g., para $x>$ 0 tem-se que $C^{\mathcal{L}}(x, \infty)-C^{\mathcal{L}}(x,-\infty)=c_{1} x+c_{5} x=x$, o que implica em $c_{1}+c_{5}=1$. As outras restrições implicam $c_{3}+c_{7}=1, c_{2}+c_{8}=1$ e $c_{4}+c_{6}=1$. Desta maneira, é possível reescrever a equação acima como:

$$
\begin{aligned}
C^{\mathcal{L}}(x, y) & =C^{\mathcal{L}++}\left(c_{1}|x|, c_{2}|y|\right) \mathbf{1}_{\{x \geq 0, y \geq 0\}} \\
& +C^{\mathcal{L}--}\left(c_{3}|x|, c_{4}|y|\right) \mathbf{1}_{\{x \leq 0, y \leq 0\}} \\
& -C^{\mathcal{L}+-}\left(\left(1-c_{1}\right)|x|,\left(1-c_{4}\right)|y|\right) \mathbf{1}_{\{x \geq 0, y \leq 0\}} \\
& -C^{\mathcal{L}-+}\left(\left(1-c_{3}\right)|x|,\left(1-c_{2}\right)|y|\right) \mathbf{1}_{\{x \leq 0, y \geq 0\}}
\end{aligned}
$$

que define uma cópula de Lévy se as constantes $c_{1}, c_{2}, c_{3}, c_{4} \in[0 ; 1]$.

Vamos interpretar esta construção. As medidas de Lévy marginais são denotadas por $\nu_{1}$ e $\nu_{2}$. Olhamos cada membro do lado direito da equação (25) originando uma medida de Lévy separadamente. A integral caudal do primeiro quadrante é

$$
U^{++}(x, y)=C^{\mathcal{L}++}\left(c_{1} U_{1}^{+}(x), c_{2} U_{2}^{+}(y)\right)
$$

Isto corresponde a uma medida de Lévy associada a um processo de Lévy de saltos positivos. Suas medidas de Lévy marginais são $c_{1} \nu_{1}(d x) \mathbf{1}_{\{x>0\}} \mathrm{e}$ $c_{2} \nu_{2}(d y) \mathbf{1}_{\{y>0\}}$. Os outros quadrantes são vistos de maneira análoga. Dessa forma, vemos que a cópula apresentada pela equação (25) é a soma de quatro partes independentes, cada uma delas correspondendo a um quadrante do domínio da medida de Lévy. A componente referente ao primeiro quadrante regula apenas os saltos positivos de $x$ e positivos de $y$ com as marginais dadas acima e a estrutura de dependência dada por $C^{\mathcal{L}++}$. A segunda parte independente referente ao segundo quadrante regula os saltos negativos de $x$ e positivos de $y$. Suas marginais são dadas por $\left(1-c_{3}\right) \nu_{1}(d x) \mathbf{1}_{\{x<0\}}$, e $\left(1-c_{2}\right) \nu_{2}(d y) \mathbf{1}_{\{y>0\}}$. Sua estrutura de dependência é regulada pela cópula de Lévy positiva $C^{\mathcal{L}-+}$. As outras duas partes independentes relativas ao terceiro e quarto quadrantes são interpretadas de maneira análoga.

A cópula apresentada na equação (25) é denominada cópula de Lévy de proporções constantes porque a proporção de saltos nos dois componentes correlacionados não depende do tamanho dos saltos (apenas do seu sinal). Estas últimas estão longe de representar o universo de todas a cópulas, porém elas são numerosas o suficiente para satisfazerem a maioria das aplicações práticas. Somado a isso, as cópulas de Lévy de processos estáveis sempre podem ser representadas pela forma dada pela equação (25).

Disponibilizamos no Apêndice as extensões necessárias para as cópulas de Lévy generalizadas multivariadas. 


\section{Aplicações}

Apresentamos uma forma de aplicação da cópula de Lévy. Como no caso das cópulas ordinárias, a escolha apropriada da cópula de Lévy permite distinguir a dependência linear de outras formas de dependências para modelar os log-retornos de ativos financeiros. Esta distinção possui aplicações em modelos de gestão de risco, onde os movimentos conjuntos de saltos negativos nos log-retornos de ativos financeiros são importantes.

Incialmente, a cópula ordinária foi sugerida como um instrumento viável para modelar opções europérias que dependem de mais de um ativo objeto, também denominadas opções multivariadas (vide Cherubini et alii (2004, capítulo 8)). Basicamente existem dois tipos de contratos de opções multivariadas. O primeiro trata de uma cesta de ativos, isto é, o ativo objeto $\tilde{S}_{T}$ é uma carteira, que pode ser representada por

$$
\begin{aligned}
\tilde{S}_{T} & =\sum_{i=1}^{N} \alpha_{i} S_{T}^{i} \\
\text { sujeito à } \sum_{i=1}^{N} \alpha_{i} & =1 \text { e } \alpha_{i} \geq 0
\end{aligned}
$$

$S^{i}, i=1, \ldots, N$ são os ativos da carteira que apresentam dependências entre si e $T=$ data de exercício do artigo-objeto.

O outro contrato trata de uma escolha de performance, ou seja, o ativo objeto é o ativo que atingiu a melhor performance durante o prazo de validade do contrato, i.e.,

$$
\tilde{S}_{T}=S_{T}^{\varphi}
$$

onde

$$
\varphi=\arg \max _{1 \leq i \leq N}\left(\frac{S_{T}^{i}}{S_{0}^{i}}\right) \text { ou } \varphi=\arg \min _{1 \leq i \leq N}\left(\frac{S_{T}^{i}}{S_{0}^{i}}\right)
$$

O parâmetro $\varphi$ tem como objetivo identificar o ativo que obteve o resultado ótimo, e dependendo do tipo de contrato, i.e., se tratar-se de opção de compra (call) ou de venda (put), este argumento pode maximizar ou minimizar a performance. Entretanto, a utilização de cópulas ordinárias para apreçar opções que dependem da trajetória (path-dependent) de $\widetilde{S}$ é inadequada, pois estes contratos dependem da informação em toda a trajetória do processo estocástico que gera os preços dos ativos em análise (DGPs). O conhecimento da estrutura de dependência dos ativos no instante terminal do processo não é suficiente para mensurar o preço do contrato adequadamente. Segue abaixo a função de ganhos e perdas no vencimento $H_{T}$ de algumas opções enquadradas na classe de dependentes das trajetórias: 
1. opção lookback de compra padrão

$$
H_{T}=\tilde{S}_{T}-\min _{0 \leq t \leq T}\left(\tilde{S}_{t}\right)
$$

2. opção lookback de venda padrão

$$
H_{T}=\max _{0 \leq t \leq T}\left(\tilde{S}_{t}\right)-\tilde{S}_{T}
$$

3. opção lookback de compra sobre o máximo

$$
H_{T}=\max \left\{\max _{0 \leq t \leq T}\left(\tilde{S}_{t}\right)-K, 0\right\}
$$

4. opção lookback de venda sobre o mínimo

$$
H_{T}=\max \left\{K-\min _{0 \leq t \leq T}\left(\tilde{S}_{t}\right), 0\right\}
$$

5. opção asiática de compra sobre a média do preço do ativo-objeto

$$
H_{T}=\max \left\{\langle\tilde{S}\rangle_{T}-K, 0\right\}
$$

6. opção asiática de venda sobre a média do preço do ativo-objeto

$$
H_{T}=\max \left\{K-\langle\tilde{S}\rangle_{T}, 0\right\}
$$

7. opção asiática de compra sobre a média do preço de exercício

$$
H_{T}=\max \left\{\tilde{S}_{T}-\langle\tilde{S}\rangle_{T}, 0\right\}
$$

8. opção asiática de venda sobre a média do preço de exercício

$$
H_{T}=\max \left\{\langle\tilde{S}\rangle_{T}-\tilde{S}_{T}, 0\right\}
$$

onde $\tilde{S}$ representa o ativo objeto que pode depender de mais de um ativo, $K$ o valor de exercício, $\langle\tilde{S}\rangle_{T}$ a média do ativo objeto ao longo do tempo até o instante $T$. Existem duas maneiras de calcular esta média, em detalhes, aritmeticamente $\langle\tilde{S}\rangle_{T}=T^{-1} \sum_{t=0}^{T} \tilde{S}_{t}$ ou geometricamente, $\langle\tilde{S}\rangle_{T}=\left(\prod_{t=0}^{T} \tilde{S}_{t}\right)^{T^{-1}}$. 
O preço do derivativo $V_{t}$ será dado pela expressão

$$
V_{t}=\mathbb{E}^{\mathbb{Q}}\left[h\left(\frac{S_{T}}{B_{T}}\right) \mid \mathcal{F}_{t}\right]
$$

onde $H_{T}$ não necessariamente está limitado as formas apresentadas nas equações (30) até (37), e, $r_{f}$ representa a taxa livre de risco.

Observe que a esperança do lado direito da equação (38) é realizada na medida neutralizadora do risco, isto é, na medida $\mathbb{Q} .{ }^{1}$ Diferentemente da medida $\mathbb{P}$, que pode ser estimada objetivamente ou subjetivamente por meio de dados históricos, a medida $\mathbb{Q}$ é medida sintética, ou seja, é construída por algum modelo estatístico de modo a possuir a propriedade que, sob ela, o processo do ativo objeto divido pelo numerário $\left\{S_{t} / B_{t}\right\}_{t>0}$ é um martingal. ${ }^{2}$

Podemos interpretar a propriedade martingal de um processo como, dada toda a informação disponível em $\mathcal{F}_{t}$, ela determina que o melhor previsor do preço no futuro é o preço de hoje. Entretanto, seria interessante que a informação contida na medida $\mathbb{P}$ contribua para a construção de $\mathbb{Q}$, tornando o modelo descrito pela equação (38) economicamente interpretável.

A derivada de Radon-Nikodym $d \mathbb{Q} / d \mathbb{P}$ (vide Shiryaev (1999, p.434)) nos permite calcular a esperança no lado direito da equação (38) na medida observada $\mathbb{P}$, como segue:

$$
V_{t}=\mathbb{E}^{\mathbb{P}}\left[\frac{d \mathbb{Q}}{d \mathbb{P}} h\left(\frac{S_{T}}{B_{T}}\right) \mid \mathcal{F}_{t}\right]
$$

Para que $d \mathbb{Q} / d \mathbb{P}$ faça sentido, as medidas $\mathbb{P}$ e $\mathbb{Q}$ devem ser equivalentes ${ }^{3}$, ou seja, a medida $\mathbb{Q}$ não deve atribuir probabilidade zero para nenhum evento (conjunto) onde $\mathbb{P}$ atribua probabilidade positiva, e vice e versa. Isto significa se existe a possibilidade de ocorrencias de eventos adversos ou benéficos, eles devem contribuir para a formação do preço $V_{t}$, o que não ocorre se atribuir probabilidade zero a estes eventos sob a medida $\mathbb{Q}$.

\footnotetext{
${ }^{1}$ Devido as propriedades de $\mathbb{Q}$ alguns autores a denominam de medida EMM, ou medida martingal equivalente.

${ }^{2}$ Podemos definir um processo martingal como:

Definição 30 Seja $\left\{X_{t}\right\}_{t>0}$ um processo de Lévy definido no espaço de probabilidade filtrado $\left(\Omega,\left\{\mathcal{F}_{t}\right\}_{t \geq 0}, \mathbb{P}\right)$. Dizemos que $\left\{X_{t}\right\}_{t \geq 0}$ é um processo martingal em relação a medida medida

onde $\Delta t>0$.

$$
\mathbb{E}^{\mathbb{P}}\left[X_{t+\Delta t} \mid \mathcal{F}_{t}\right]=X_{t}, \text { q.c. }
$$

${ }^{3}$ Podemos definir medidas equivalentes como:

Definição 31 Sejam $\nu$ e $\eta$ duas medidas de Lévy definidas no espaço mensurável $(\Omega, \mathcal{F})$ e $A \subset \mathcal{F}$ um conjunto não vazio. Dizemos que $\nu$ e $\eta$ são medidas equivalentes, ou simplesmente, $\nu \sim \eta$, se e somente se, para todo $A \subset \mathcal{F}$,
}

$$
\nu(A)=0 \Longleftrightarrow \eta(A)=0
$$


Sem muita formalização, temos que o Teorema Fundamental de Apreçamento de Ativos (vide Shiryaev (1999, p.413)) nos diz que a existência de uma medida $\mathbb{Q}$ garante as propriedades de não arbitragem, ${ }^{4}$ e pelo Segundo Teorema Fundamental de Apreçamento de Ativos, (vide Shiryaev (1999, p.481)) temos que a unicidade da medida $\mathbb{Q}$ implica completitude do mercado, ou seja, existe um único preço livre de arbitragem. Desta maneira, caso exista mais de uma medida $\mathbb{Q}$ equivalente a $\mathbb{P}$ então não teremos um preço único, mas um intervalo de valores de preços que o derivativo poderá assumir. O preço que será praticado no mercado, dependerá, assim, das preferências (propriedades das funções de utilidades) dos agentes financeiros.

Esta questão pode ser resolvida estimando-se a derivada de Radon-Nykodim $d \mathbb{Q} / d \mathbb{P}$. Fajardo e Mordecki (2006) abordaram a questão da mudança de medida no contexto bivariado. A metodologia sugerida por eles permite o desenvolvimento de fórmulas analíticas para alguns tipos de opções, tais como: opção de inadimplência, opções de Margrabe, opções de swap, e, opções quanto. Entretanto, esta metodologia está fundamentada na hipótese que a função de payoffs $h$ do derivativo é homogênea nos ativos-objetos, o que limita a sua aplicação.

Desta maneira, seguiremos a abordagem mais comum, proposta por Gerber e Shui (1994), que utiliza a transformada de Esscher e permite nos lidar de maneira mais objetiva com a questão da existência de um preço livre de arbitragem, conforme é mostrado pelo seguinte teorema:

Teorema 32 Seja $\left\{X_{t}\right\}_{0 \leq t \leq T}$ um processo de Lévy definido no espaço de probabilidade filtrado $\left(\Omega,\left\{\mathcal{F}_{t}\right\}_{0 \leq t \leq T}, \mathbb{P}\right)$ e caracterizado por pela tripla $(\gamma, \Sigma, \nu)$. Para um $\theta \in \mathbb{R}$, se

$$
\mathbb{E}^{\mathbb{P}}\left[e^{\theta\left(X_{T}\right)}\right]<\infty
$$

então

$$
\frac{d \mathbb{P}^{\theta}}{d \mathbb{P}}=\frac{e^{\theta X_{T}}}{\mathbb{E}^{\mathbb{P}}\left[e^{\theta X_{T}}\right]}=e^{\theta X_{T}-\varphi(\theta)(T-t)}
$$

onde $\mathbb{E}^{\mathbb{P}}\left[e^{\theta X_{T}}\right]=e^{\varphi(\theta)(T-t)}, \mathbb{E}^{\mathbb{P}}\left[d \mathbb{P}^{\theta} / d \mathbb{P}\right]=1$, e $\mathbb{P}^{\theta}$ define a medida de probabilidade, tal que $\mathbb{P} e \mathbb{P}^{\theta}$ são equivalentes, $e\left\{X_{t}\right\}_{t \geq 0}$ sob a medida $\mathbb{P}^{\theta}$ é caracterizado pela tripla $\left(\gamma^{\theta}, \Sigma^{\theta}, \nu^{\theta}\right)$ dado

$$
\gamma^{\theta}=\gamma+\theta \Sigma+\int_{-\infty}^{\infty}\left(e^{\theta \cdot x}-1\right) x \mathbf{1}_{\{|x| \leq 1\}} \nu(d x)
$$

${ }^{4}$ Mais tecnicamente, no contexto dos processos de Lévy, estes devem ser $\sigma$-martingais e a existência de uma medida $\mathbb{Q}$ que atenda esta propriedade garante a condição de NFLVR (No Free Lunch with Vanishing Risk), isto é, não existe almoço grátis quando o risco se estingüe, condição equivalente a de não arbitragem. Para maiores detalhes vide Delbaen e Schachermayer (1998). 


$$
\Sigma^{\theta}=\Sigma
$$

$$
\nu^{\theta}(d x)=e^{\theta x} \nu(d x)
$$

A prova deste teorema pode ser encontrada em Hubalek e Sgarra (2006). A condição descrita pela equação (40) garante a existência de uma medida $\mathbb{P}^{\theta}$ equivalente a $\mathbb{P}$, e, pelo Teorema Fundamental de Apreçamento de Ativos, garantimos que o preço será livre de arbitragem (não existe almoço grátis). a transformada de Esscher é descrita pela equação (41) e possui a propriedade de alterar não só a tendendêcia determinística $\gamma$ como também a medida de Lévy $\nu$. Observe que o componente que descreve o processo de difusão $\Sigma$, não se altera, sendo que o Teorema de Girsanov torna-se um caso particular desta transformada, correspondendo ao caso quando $\nu=0$

Entretanto, o processo, sob à medida equivalente $\mathbb{P}^{\theta}$, não é necessariamente um martingal. Assim, conforme é apontado em Nelsen (1999, equação 33), ou Shiryaev (1999, p. 698), devemos introduzir a condição adicional

$$
\varphi(\theta+1)-\varphi(\theta)=0
$$

e se seguirmos a mesma linha de raciocínio da equação (40), necessitamos também que

$$
\mathbb{E}^{\mathbb{P}}\left[e^{\left(\varphi(\theta+1)-r_{f}\right)(T-t)}\right]<\infty
$$

Resolver a equação (45), significa obter as raízes de um polinômio, que em geral não são iguais. Desta maneira, quando houver duas ou mais raízes distintas temos pelo Segundo Teorema Fundamental de Apreçamento de Ativos que o mercado é incompleto, ou seja, haverá mais de um preço livre de arbitragem sendo negociado pelos agentes de mercado.

Existem algumas abordagens para lidar com a incompletude de mercado, entretanto, não nos aprofundaremos na discussão para não fugir do escopo deste trabalho. Apenas para ilustrar, podemos citar os seguintes critérios:

- medida martingal de mínima entropia (vide Frittelli (2000)):

$$
\mathbb{Q}=\arg \min I(\mathbb{Q}, \mathbb{P})
$$

onde $I$ é a entropia relativa (vide Cover e Thomas (1991)) entre $\mathbb{Q}$ e $\mathbb{P}$, que mede o quanto a distribuição $\mathbb{Q}$ diverge em relação a $\mathbb{P}$, e pode ser expressa como:

$$
I(\mathbb{Q}, \mathbb{P})= \begin{cases}\mathbb{E}^{\mathbb{P}}\left[\frac{d \mathbb{Q}}{d \mathbb{P}} \log \left(\frac{d \mathbb{Q}}{d \mathbb{P}}\right)\right], & \text { se } \mathbb{P}>0 \\ +\infty, & \text { se } \mathbb{P}=0\end{cases}
$$


- medida martingal do máximo preço (vide Karoui e Quenez (1995)):

$$
\mathbb{Q}=\arg \max \mathbb{E}^{\mathbb{Q}}\left[h\left(\frac{S_{T}}{B_{T}}\right) \mid \mathcal{F}_{t}\right]
$$

Este critério avalia o preço sob à perspectiva do vendedor de um derivativo, visando, assim, obter o preço máximo.

- medida martingal de máxima utilidade (vide Karoui e Rouge (2000)):

$$
\mathbb{Q}=\arg \max \mathbb{E}^{\mathbb{Q}}\left[u\left\{h\left(\frac{S_{T}}{B_{T}}\right)\right\} \mid \mathcal{F}_{t}\right]
$$

onde $u$ é uma função de utilidade que orientará o comportamento do investidor, ou agente representativo do mercado. Este critério avalia o preço sob a perspectiva do comprador, ou seja, o comprador quer maximizar a sua satisfação ao comprar um intrumento financeiro.

\subsection{Modelo do log-retorno}

É fácil observar que os ganhos e perdas destes contratos dependem de toda informação da trajetória. Neste caso, a cópula de Lévy é o instrumento adequado, pois ela é capaz de modelar toda a informação desejada. Para aplicar a teoria apresentada no apreçamento dos contratos acima mencionados, vamos assumir que as hipóteses referentes aos processos de de Lévy são válidas, ou seja, temos estacionariedade, incrementos independentes e continuidade estocástica. Note que as duas primeiras hipóteses podem ser testadas, respectivamente, por testes de raízes unitárias (vide Maddala e Kim (1999)), e pelo BDS (vide Brock et alii (1996)). Seja o processo do log-retorno dado pelo modelo

$$
Y_{t}^{i}=\ln \left(\frac{S_{t}^{i}}{S_{t-1}^{i}}\right)=X_{t}^{i, u}-X_{t}^{i, d}
$$

onde $X_{t}^{i, u}$ é um processo gama do ativo $i$, que orientará o comportamento dos saltos positivos $(u)$ e $X_{t}^{i, d}$ um processo gama que orientará os saltos negativos $(d)$. O processo gama é um processo da classe de Lévy, onde a medida de Lévy é dada por

$$
\nu_{i}^{u, d}(x)=\frac{c_{i}^{u, d} \exp \left\{-\lambda_{i}^{u, d} x\right\}}{x} \mathbf{1}_{\{x>0\}}
$$

onde $\lambda_{i}^{u, d}>0$ determina a taxa de decaimento dos saltos, ou seja, a probabilidade de ocorrerem saltos grandes, e $c_{i}^{u, d}>0$ determina a freqüência dos saltos, sejam pequenos ou grandes, (veja Cont e Tankov (2004)). O modelo é colocado como a diferença de dois processos gamas, haja vista que processo gama é um subordinador e possui apenas saltos positivos. Adicionalmente, e sem perda de generalidade, 
o modelo assume que $\lambda_{i}^{u, d}=\tilde{\lambda}$, isto é, as freqüências de saltos positivos e negativos por unidade de tempo são iguais. Fizemos estas escolhas por conveniência e para facilitar a ilustração da teoria apresentada.

\subsection{Estimação}

Existem várias abordagens econométricas para estimar um modelo, destre as quais as mais frequentemente utilizadas em finanças são Máxima Verossimilhança (MLE) e Método Generalizado dos Momentos (GMM). O GMM é o método mais robusto, quando comparado com o MLE, sendo preferível sob a perspectiva dos econometristas. A necessidade da robustez ocorre por não se conhecer a verdadeira distribuição que gera ou produz os log-retornos das séries financeiras. Esta robustêz tem como custo a perda da eficiência do estimador quando se considera o critério do erro quadrático mínimo (MSE). Entretanto, esta perda de eficiência não prejudica a consistência do estimador GMM, mesmo na condição de especificação incorreta do modelo (distribuição dos log-retornos). Por outro lado, o método MLE gera estimadores eficientes, considerando-se o critério MSE. Entretanto, erros na especificação do modelo podem afetar seriamente a consistência do estimador, qualidade indispensável para as aplicações em finanças. Adicionalmente, quando o número de observações é relativamente grande, como é o caso das séries diárias de log-retornos, o problema de eficiência passa a ser secundário, pois utilizamos, em tal situação, as propriedades assintóticas dos estimadores.

Outra questão a ser levantada é que para muitas distribuições não-gaussianas produzidas por atividade infinita de saltos de um processo de Lévy podem não apresentar expressões analíticas que permitam calcular a função de máxima verossimilhança, o que inviabilizaria a utilização do método MLE. Em contrapartida, pelo método GMM, podemos obter estimativas dos parâmetros fazendo uso de um número finito de momentos, o que é sempre possível obter por meio dos dados observados. Dado estas considerações, fica evidente que as vantagens do GMM quando comparado com o MLE, desta maneira adotaremos o método GMM em nossas aplicações.

Considerando o modelo descrito na subseção anterior, o processo do log-retorno apresentará incrementos ostentando a seguinte pdf (função de densidade de probabilidade) sob a medida $\mathbb{P}$ :

$$
Y_{\Delta t}^{i} \sim p_{\Delta t}^{i, u}\left(x_{u}\right) p_{\Delta t}^{i, d}\left(x_{d}\right), x_{u}>0 \text { e } x_{d}>0
$$

onde

$$
p_{\Delta t}^{i, u, d}=\frac{\tilde{\lambda}_{i}^{\left(c^{u, d} \Delta t\right)}}{\Gamma\left(c_{i}^{u, d} \Delta t\right)} x_{u, d}^{\left(c_{i}^{u, d} \Delta t\right)-1} \exp \left\{-\tilde{\lambda}_{i} x_{u, d}\right\}
$$

e $\Gamma(\cdot)$ é a função gama. Como decorrência, temos os seguintes momentos-centrados: 


$$
\begin{aligned}
\mu_{0, \Delta t}^{i} & =\left(\frac{c_{i}^{u}-c_{i}^{d}}{\tilde{\lambda}_{i}}\right) \Delta t \\
\mu_{2, \Delta t}^{i} & =\left(\frac{c_{i}^{u}+c_{i}^{d}}{\tilde{\lambda}_{i}^{2}}\right) \Delta t \\
\mu_{3, \Delta t}^{i} & =2\left(\frac{c_{i}^{u}-c_{i}^{d}}{\tilde{\lambda}_{i}^{3}}\right) \Delta t \\
\mu_{4, \Delta t}^{i} & =3\left[\frac{\left(c_{i}^{u}+c_{i}^{d}\right)^{2} \Delta t+2\left(c_{i}^{u}+c_{i}^{d}\right)}{\tilde{\lambda}_{i}^{4}}\right] \Delta t
\end{aligned}
$$

Temos que $\Delta t$ representa a unidade de medida do tempo, que em geral, na prática, é um dia útil (base 252). Também, usualmente, são válidas as seguintes propriedades $c_{i}^{u, d}(T)=T c_{i}^{u, d}(\Delta t)$ e $\tilde{\lambda}_{i}(T)=\tilde{\lambda}_{i}(\Delta t)$. Observe que o parâmetro $\tilde{\lambda}_{i}$ não é influenciado sobre a mudança de escala, ou seja, probabilidade de saltos grandes continua a mesma. Por outro lado, $c_{i}^{u, d}$ depende do tempo, i.e., a frequência dos saltos é diretamente proporcional ao tempo. Assim, mesmo que $\tilde{\lambda}_{i}$ não dependa do tempo, a medida que $T$ aumenta, uma maior quantidade de saltos ocorrem, e consequentemente, espera-se, uma maior observação de saltos grandes. Levando em consideração os resultados analíticos para os momentos de $Y_{t}^{i}$, é relativamente fácil estimar os parâmetros dos processos dos log-retornos por meio do método GMM (vide Hall (2005)), que pode ser expresso como

$$
\arg \min _{\theta^{i}}\left(g^{i}\left(\zeta^{i}, Y_{t}^{i}\right)\right)^{\top t}\left(W^{i}\right)^{-1} g^{i}\left(\zeta^{i}, Y_{t}^{i}\right)
$$

onde $\zeta^{i}=\left(c_{i}^{u}, c_{i}^{d}, \tilde{\lambda}_{i}\right) \mathrm{e}$

$$
g^{i}\left(\zeta^{i}, Y_{t}^{i}\right)=\left[\begin{array}{c}
T^{-1} \sum_{t=1}^{T} Y_{t}-\mu_{0, \Delta t}^{i}\left(\zeta^{i}\right) \\
T^{-1} \sum_{t=1}^{T}\left(Y_{t}-T^{-1} \sum_{t=1}^{T} Y_{t}\right)^{2}-\mu_{2, \Delta t}^{i}\left(\zeta^{i}\right) \\
T^{-1} \sum_{t=1}^{T}\left(Y_{t}-T^{-1} \sum_{t=1}^{T} Y_{t}\right)^{3}-\mu_{3, \Delta t}^{i}\left(\zeta^{i}\right) \\
T^{-1} \sum_{t=1}^{T}\left(Y_{t}-T^{-1} \sum_{t=1}^{T} Y_{t}\right)^{4}-\mu_{\Delta t}^{i}\left(\zeta^{i}\right)
\end{array}\right]
$$

A matriz de pesos $W^{i}$ pode ser estimada pelo procedimento sugerido por Newey-West (vide Newey e West (1987)) utilizando o truncamento que depende do tamanho da amostra para determinar quantas matrizes defasadas de covariâncias devem ser incluídas no procedimento, no caso, a parte inteira de $4(100 T)^{2 / 9}$.

Note que os parâmetros estimados por GMM são obtidos por meio da equação (52), que envolve necessariamente algum método de otimização, o que pode envolver questões numéricas. Entretanto, se houverem restrições impostas aos parâmetros a serem estimados, o ponto de partida ou solução inicial do problema de 
otimização pode não atingir um mínimo global, ou seja, os parâmetros estimados representam uma solução subótima, i.e., um mínimo local.

Para exemplificar, considere as séries temporais diárias dos log-retornos das ações Petrobrás PN (ticker: PETR4 e $i=1$ ) e Vale do Rio Doce PNA N1 (ticker: VALE5 e $i=2$ ), para o período de 2.01.2002 até 2.10.2008, ou seja, uma amostra de tamanho $T=1533$ e intervalo de tempo $\Delta t=1$ (dia útil). Para termos certeza que os parâmetros obtidos fossem resultantes de um mínimo global, utilizamos a função Minimize do Mathematica 6.0, evitando a questão do parágrafo anterior. Esta abordagem produziu as seguintes estimativas para os parâmetros do modelo sob a medida $\mathbb{P}: c_{1}^{u}=1.28947, c_{1}^{d}=1.17862, \tilde{\lambda}_{1}=1.73504, c_{2}^{u}=3.74614$, $c_{2}^{d}=3.50189$, e $\tilde{\lambda}_{2}=2.96173$.

Uma questão que surge é como realizar a mudança de medida, ou seja, como obter uma medida martingal $\mathbb{Q}$ equivalente $\mathbb{P}$. A solução está em estimar a derivada de Radon-Nykodim por meio da transformada de Esscher, conforme abordagem sugerida na subseção anterior (equações (41) e (45)). Observe que as condições, apresentadas pelas equações (40) e (46), são satisfeitas vis-à-vis a pdf apresentada na equação (50), para $\tilde{\lambda}_{i}>\theta_{i}$, produzindo o seguinte resultado

$$
\mathbb{E}^{\mathbb{P}}\left[e^{\theta_{i}\left(Y_{\Delta t}^{i}-r_{f}\right)}\right]=e^{\theta_{i} r_{f}} \tilde{\lambda}_{i}^{\left(c_{i}^{u}+c_{i}^{d}\right) \Delta t}\left(\tilde{\lambda}_{i}-\theta_{i}\right)^{-c_{i}^{u} \Delta t}\left(\tilde{\lambda}_{i}+\theta_{i}\right)^{-c_{i}^{d} \Delta t}<\infty
$$

Assim, garantimos a existência da medida martingal e de um preço livre de arbitragem (Teorema Fundamental de Apreçamento de Ativos). Por outro lado, pela equação (45) temos uma única raíz finita, i.e., $\theta_{1}=-0.48407$ e $\theta_{2}=-0.50384$, que atendem a condição $\tilde{\lambda}_{i}>\theta_{i}$. Desta maneira, temos um mercado completo $(\mathrm{Se}$ gundo Teorema Fundamental de Apreçamento de Ativos) e o preço do derivativo $V_{t}$ poderá ser obtido pela equação (39), onde

$$
\frac{d \mathbb{Q}}{d \mathbb{P}}=e^{\theta_{i} Y_{\Delta t}-\varphi_{i}\left(\theta_{i}\right) \Delta t}=\frac{1}{\Delta t} \log \mathbb{E}^{\mathbb{P}}\left[e^{\theta Y_{\Delta t}^{i}}\right]
$$

\subsection{Simulação de Monte Carlo}

Conhecendo os parâmetros que descrevem o processo de Lévy, isto é a tripla $(\gamma, \Sigma, \nu)$, sob a medida $\mathbb{Q}$, que podem ser obtidos pelo procedimento sugerido na subseção anterior, podemos, por meio do método de simulação de Monte Carlo calcular numericamente os preços dos derivativos discutidos neste artigo. Utilizamos o procedimento sugerido Rosinski para gerar os processos gama (vide Rosinski (2001)). Adaptamos os algorítmos para que seja possível simular os processos de Lévy para qualquer unidade de tempo, i.e., fazemos,

$$
X_{t}^{i, u, d}=\sum_{j=1}^{n(j)}\left(\lambda_{i}^{u, d}\right)^{-1} \exp \left\{-\frac{\Gamma_{i, j}^{u, d}}{T c_{i}^{u, d}}\right\} V_{i, j}^{u, d} \mathbf{1}_{\left\{U_{i, j}^{u, d} \leq t\right\}}
$$


onde $c_{i}^{u d}$ e $\lambda_{i}^{u, d}$ são os parâmetros do processo gama medidos para uma unidade de tempo, $T$ é o valor terminal(vencimento do contrato), $\left\{U_{i, j}^{u, d}(0, T)\right\}_{j=1, \ldots, n(j)}$ e $\left\{V_{i, j}^{u, d}(T)\right\}_{j=1, \ldots, n(j)}$ são seqüências I.I.D. de valores observados, respectivamente, de uma distribuição uniforme no intervalo $[0, T]$ e de uma distribuição exponencial com parâmetro $T . \Gamma_{i, j}^{u, d}=\sum_{z=1}^{j} W_{i, z}^{u, d}$, onde $\left\{W_{i, z}^{u, d}(1)\right\}_{z=1, \ldots, n(j)}$ é uma seqüência I.I.D. de valores obtidos de uma distribuição exponencial padronizada e $n(j)=\sup \left\{j: \Gamma_{i, j}^{u, d} \leq \tau\right\}$ é o truncamento da somatória que teoricamente deveria ser indexada em todo conjunto dos naturais. O truncamento $n(j)$ depende do parâmetro $\tau$ que determinará a precisão do processo. Quanto maior for $\tau$ teremos uma maior quantidade de termos $\Gamma_{i, j}^{u, d}$ influenciando o processo. Como $\Gamma_{i, j}^{u, d}$ é um processo estritamente crescente, a medida que $j \longrightarrow \infty$, temos que $\Gamma_{i, j}^{u, d} \longrightarrow \infty$ e, consequentemente, $\exp \left(-\Gamma_{i, j}^{u, d}\right) \longrightarrow 0$. Assim, como $\tau$ limita os valores aceitáveis de $j$, então significa que ele define a partir de qual magnitude os saltos passam a ser desprezados para compor $X_{t}^{i, u, d}$.

O procedimento de Rosinski refere-se exclusivamente a séries univariadas marginais, sendo necessário, portanto, incluir uma estrutura da cópula de Lévy para que se possa gerar as séries multivariadas. Consideramos aqui apenas o caso bivariado, i.e., $i=1,2$.

O parâmetro $\Gamma_{2, j}^{u, d}$ pode escrito como uma função de $\Gamma_{1, j}^{u, d}$, por meio da função inversa da equação (17), i.e.,

$$
\Gamma_{2, j}^{u, d}=\left(C_{\Gamma_{1, j}^{u, d}}^{\mathcal{L}}\right)^{-1}\left(F_{j}^{u, d}\right)
$$

onde $F_{j}^{u, d}$ representa uma distribuição acumulada, ou seja, $\left\{F_{j}^{u, d}\right\}_{j=1, \ldots, n(j)}$ é uma seqüência I.I.D. de variáveis uniformes padronizadas. Note que neste ponto estamos introduzindo a dependência entre os processos. Aplicando as cópulas de Clayton (equação 14) e de Frank (equação 15), temos, respectivamente, que,

$$
\begin{aligned}
& \text { Clayton }: \quad \Gamma_{2, j}^{u, d}=\Gamma_{1, j}^{u, d}\left\{\left(F_{j}^{u, d}\right)^{-\frac{\xi^{u, d}}{1+\xi^{u, d}}}-1\right\}^{-\frac{1}{\xi^{u, d}}} \\
& \text { Frank } \quad: \quad \Gamma_{2, j}^{u, d}=\frac{1}{\xi^{u, d}} \log \left(1+\exp \left\{\xi^{u, d} \Gamma_{1, j}^{u, d}\right\}\right)\left(\frac{F_{j}^{u, d}}{1-F_{j}^{u, d}}\right)
\end{aligned}
$$

Em nossa análise foram utilizados dois softwares: Mathematica 6.0 e Matlab 7.4. Este último mostrou-se bem mais veloz na geração das séries bivariadas acima. Um procedimento de Monte Carlo com $m=10^{2}$ repetições com uma precisão $\tau=10^{3}$, custou algumas fraçoes de segundos no Matlab 7.4 num 
computador Pentium Duo 2 Core. As Figuras 1 e 2 nos permitem visualizar as diferentes estruturas das cópulas de Clayton e Frank. Consideramos os seguintes parâmetros, $c_{1}^{u, d}=c_{2}^{u, d}=\lambda_{1}^{u, d}=\lambda_{1}^{u, d}=10, m=10^{3}, T=1$, e $\tau=10^{3}$. Podemos ver claramente que a medida que os parâmetros $\theta$ das respectivas cópulas variam $\xi^{u, d}=\left(10^{-3}, 10^{-2}, 10^{-1}, 10^{0}, 10^{1}, 10^{2}, 10^{3}, 10^{4}\right)$, a estrutura de dependência entre as variáveis log-retornos dos ativos 1 e 2 assumem formas que podem afetar bastante os preços dos contratos de opções multivariados com dependências nas trajetórias.
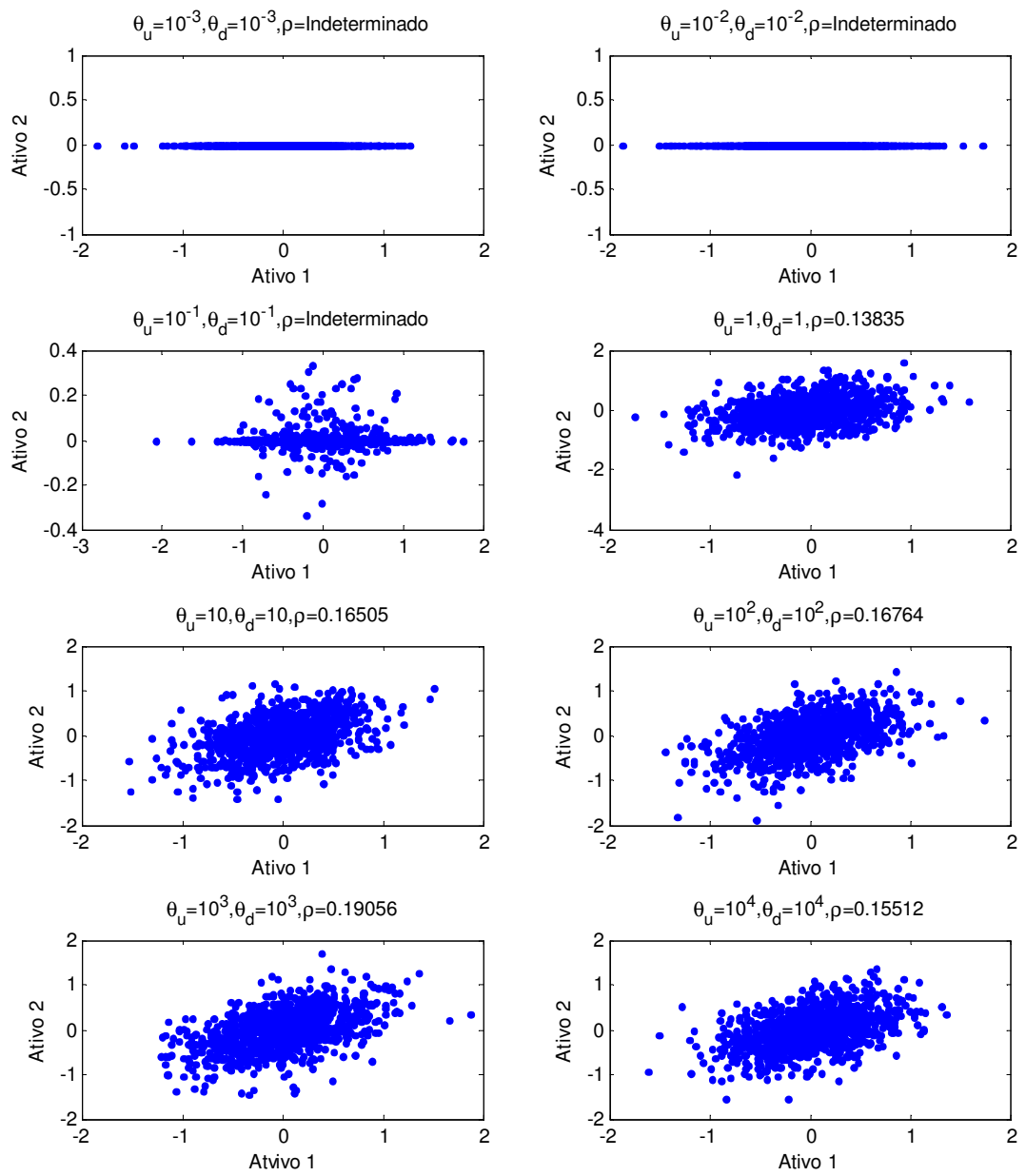

Figura 1

Análise da influência dos parâmetros da cópula de Lévy de Clayton 

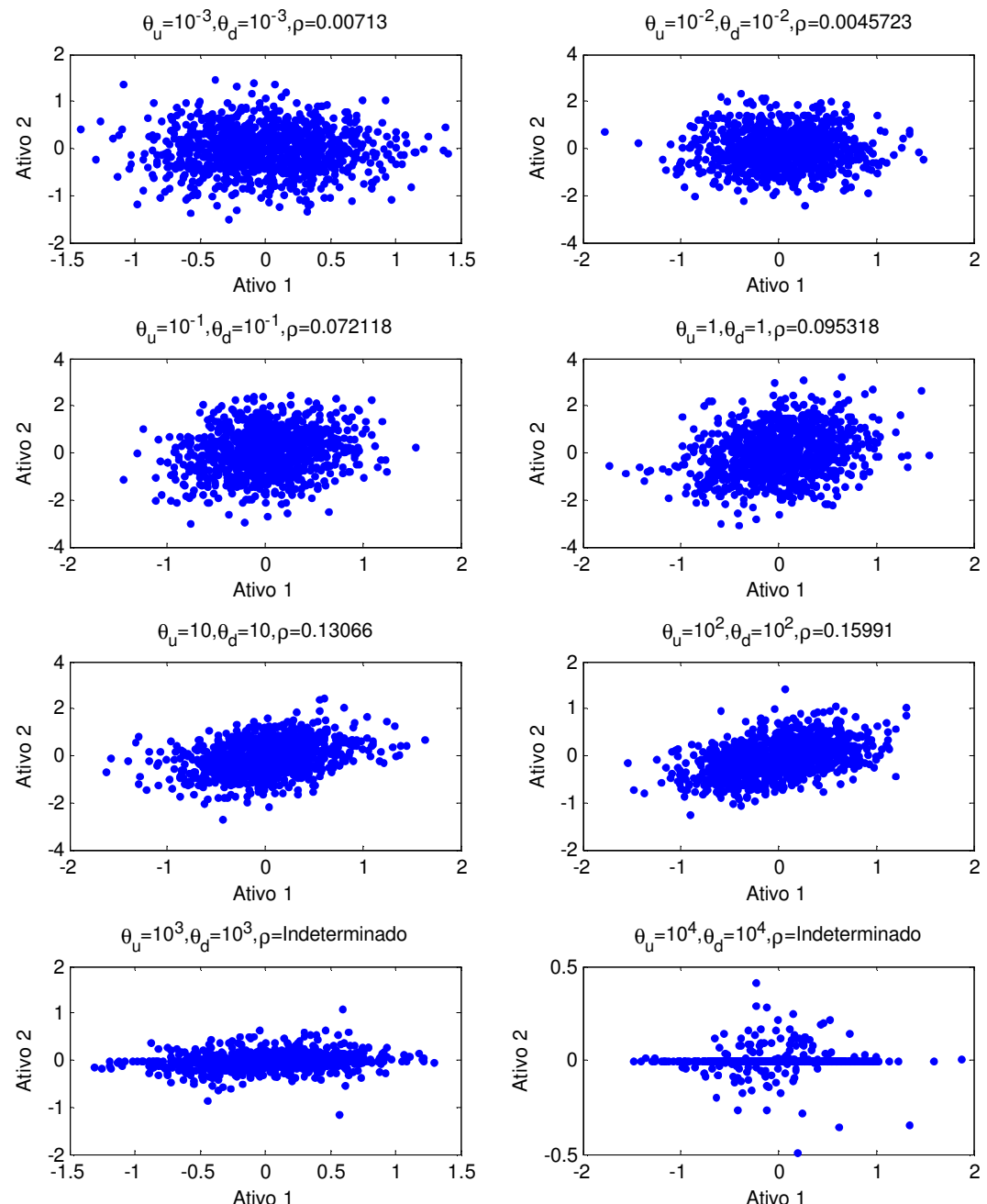

Figura 2

Análise da influência dos parâmetros da cópula de Lévy de Frank

Das opções mencionadas neste artigo, consideramos as asiáticas de compra e venda sobre a média do preço do ativo-objeto, que neste caso será uma carteira composta pelos dois ativos em análise. Observe que foram calculadas as médias aritméticas e geométricas. Os resultados apresentados na Tabela 1, referem-se aos preços das opções asiáticas de compra usando médias aritmética (AmC) e geométrica $(\mathrm{AgC})$ e das opções asiáticas de venda usando médias aritmética $(\mathrm{AmP})$ e geométrica (AgP), que são obtidos pela equação (38), onde o preço 
de cada ativo é dado por $S_{t}^{i}=S_{0}^{i} \exp \left\{Y_{t}^{i}\right\}$, para $S_{0}^{1}=40$ e $S_{0}^{2}=60$. Foram considerados ambas as cópulas (Clayton e Frank), preço de exercício $K=(20,30, \ldots, 80)$, a proporção de cada um ativo na carteira $\alpha_{1}=$ $(0,0,20, \ldots, 1)$ e $\alpha_{2}=1-\alpha_{1}, r_{f}=0,08, T=1$. Apesar da taxa livre de risco $r_{f}$ ter sido considerada de maneira determinística, isto é, constante ao longo do tempo, é relativamente fácil flexibilizar está hipótese para o contexto local ou estocástico, isto é, a taxa livre de risco é modelada por uma função determinística do tempo (local) ou por um processo estocástico com reversão a média (estocástica). Note que os preços das opções de compra diminuem a medida que o preços de exercícios aumentam e os preços das opções de venda diminuem. Em relação as cópulas, vemos que, para os parâmetros considerados, i.e., $\xi^{u, d}=10^{-1}$ para cópula de Clayton e $\xi^{u, d}=10^{3}$ para a cópula de Frank, a cópula de Frank gera distribuições menos voláteis para os log-retornos das séries, considerando uma dependência linear semelhante, i.e., a mesma autocorrelação serial do processo dos log-retornos, o que implica preços menores para as opções utilizando a cópula de Frank (vide Tabela 1). Nos casos extremos de $\alpha_{1}=0$ ou $\alpha_{1}=1$, temos que a carteira é formada apenas pelo ativo 1 e 2 respectivamente, sendo portanto, os casos de opções univariadas, casos particulares. No que se refere as médias aritméticas e geométricas, temos que as opções de compra apresentam preços maiores para as médias aritméticas e as opções de venda apresentam preços maiores para as médias geométricas. Isso se dá principalmente pelo fato da média aritmética ser sempre maior ou igual que a média geométrica, ou em outras palavras, $\langle\tilde{S}\rangle_{A} \geq\langle\tilde{S}\rangle_{G}$. Deixamos para os interessados o manuseio dos outros contratos apresentados no início desta seção, cujas complexidades são bem menores que as opções asiáticas avaliadas aqui. Por último analisamos o efeito da dependência sobre o valor das opções asiáticas considerando a média aritimética, vide Tabelas 2 e 3, onde as autocorrelações seriais do processo dos log-retornos foram obtidas considerando os parâmetros $\xi^{u, d}=10(\rho=0,187), \xi^{u, d}=0,8$ $(\rho=0,0943)$, e $\xi^{u, d}=0,5(\rho=0,0482)$ para a cópula de Clayton e $\xi^{u, d}=10^{2}$ $(\rho=0,1259), \xi^{u, d}=10(\rho=0,1068)$, e $\xi^{u, d}=1(\rho=0,0741)$ para a cópula de Frank. Note que a medida que a dependência linear diminui (autocorrelação serial do processo), temos que o preço das opções aumentam. Esse resultado vai em conformidade com os princípios de diversificação do risco idiosincrático das ações, haja vista que o ativo objeto é uma carteira de ativos. Quanto mais diversificada for esta carteira, haverá uma tendência maior de remanescer apenas o risco sistêmico, e consequiêntemente, o preço da opção atingirá o valor máximo dado uma mesma estrutura de dependência. Este último resultado deve ser analisado com bastante interesse pois ele permite uma melhoria relevante no processo gestão do risco de mercado. Os programas computacionais utilizados neste trabalho estão disponíveis no site www.ime.usp.br/nitanaka/. 


\section{Conclusões}

Uma maneira sistematizada de descrever a estrutura de dependência entre duas variáveis estocásticas é por meio de funções de cópulas. Com a necessidade de melhores instrumentos de gestão de risco, verifica-se um rápido desenvolvimento da necessidade de instrumentos financeiros mais sofisticados, capazes de modelarem derivativos que dependem não só da trajetória do processo do preço, como também, de uma quantidade finita de ativos. Neste contexto, o estudo apresentou o desenvolvimento recente da teoria de cópulas realizado principalmente por Cont e Tankov (2004) e Kallsen e Tankov (2006), que estende o conceito da cópula ordinária, que lida com um vetor aleatório, para o contexto de processos de Lévy. Passamos para a cópula de Lévy que permite mitigar de uma maneira mais eficiente o processo de precificação dos intrumentos financeiros acima citados. A construção de modelos por meio de cópulas de Lévy é uma abordagem que permite especificar a estrutura de dependência entre os log-retornos de maneira bastante flexível. Aqui apresentamos um modelo relativamente didático, considerando as suas preocupações econométricas e utilizando técnicas de estimação e de simulação de Monte Carlo. Os exemplos visaram estabelecer uma conexão entre a teoria desenvolvida e a sua aplicação ná área de apreçamento de contratos derivativos, mostrando que o método sugerido neste artigo é viável e conputacionalmente eficiente. Uma das limitações da análise da aplicação realizada no artigo é a utilização de uma medida de dependência linear (autocorrelação serial), dada a inexistência de uma medida de dependência do processo, análogo ao Tau de Kendall para variáveis aleatórias. Uma tentativa de mimicar essa medida para processos seria utilizar

$$
\tau^{\mathcal{L}}=\underset{d X_{1} d X_{2} d Y_{1} d Y_{2}-1}{2 \int_{0}^{\infty} \int_{\left\{\left(X_{2}-X_{1}\right)\left(Y_{2}-Y_{1}\right) \geq 0\right\}}^{\infty} f_{Y_{1} \mid X_{1}}^{\infty} f_{X_{1}} f_{Y_{2} \mid X_{2}} f_{X_{2}}}
$$

onde

$$
f_{Y \mid X}=\left(\frac{\partial^{2}}{\partial y^{2}} C^{\mathcal{L}}(x, y)\right)
$$

e $f_{X}(x)$ seria a distribuição marginal do log-retorno de $X$. Apesar de ser uma extensão natural do tau de Kendal ela não se concretiza pois a obtenção de soluções analíticas é relativamente difícil. 
Por último, a aplicação desta teoria também poderia ser extendida na gestão de outros riscos, sendo que o novo Acordo da Basiléia II (vide Bank of International Settlements (2005)) vem orientando as instituições financeiras a realizarem análises quantitativas de riscos de mercado, crédito, liquidez e operacional, exigindo que os modelos de risco sejam robustos às hipóteses tradicionais de mercado, principalmente em países emergentes como o Brasil, onde a economia não é considerada estável, o que significa que os modelos gaussianos nem sempre são adequados.

\section{Referências}

Bank of International Settlements (2005). International convergence of capital measurement and capital standards. Disponível em http://www.bis.org/publ/bcbs118.htmÄcesso em 15.10.2007.

Barndorff-Nielsen, O. E. (1997). Normal inverse gaussian distributions and stochastic volatility modelling. Scandinavian Journal of Statistics, 24:1-13.

Barndorff-Nielsen, O. E. (1998). Processes of normal inverse Gaussian type. Finance and Stochastics, 2:41-68.

Brock, W. A., Dechert, W. D., Scheinkman, J. A., \& LeBaron, B. (1996). A test for independence based on the correlation dimension. Econometric Reviews, 15(3):197-235.

Cherubini, U. \& Luciano, E. (2002). Bivariate options pricing with Copulas. Applied Mathematical Finance, 9(2):69-86.

Cherubini, U., Luciano, E., \& Vecchiato, W. (2004). Copulas Methods in Finance. Wiley, London.

Cont, R. \& Tankov, P. (2004). Financial Modeling with Jump Processes. Chapman $\&$ Hall/CRC, London.

Cover, T. M. \& Thomas, J. A. (1991). The Elements of Information Theory (Wiley Series in Telecomunications). Wiley, New York.

Delbaen, F. \& Schachermayer, W. (1998). The fundamental theorem of asset pricing for unbounded stochastic processes. Mathematische Annalen, 312(2):215250 .

Eberlein, E. (2001). Application of generalized hyperbolic lévy motions in finance. In: barndorff-nielsen, o. e.; mikosch, t.; resnick, s.i. (org.). Lévy Processes: theory and applications. Boston: Birkhäuser, 319-336.

Fajardo, J. \& Farias, A. (2004). Generalized hyperbolic distributions and Brazilian data. Brazilian Review of Econometrics, 24(1):1-21. 
Fajardo, J. \& Farias, A. (2007). Derivative pricing using multidimensional generalized hyperbolic distributions. Proccedings of the Seventh Brazilian Society Meeting, São Paulo.

Fajardo, J. \& Mordecki, E. (2006). Pricing derivatives on two Lévy driven stocks. International Journal od Theoretical and Applied Finance, 9(2):185-197.

Frittelli, M. (2000). The minimal entropy martingale measure and the valuation problem in incomplete markets. Mathematical Finance, 10(1):39-52.

Gerber, H. U. \& Shui, E. S. W. (1994). Option pricing by Esscher transform. Transactions of the Society of Actuaries, 46:99-191.

Hall, A. R. (2005). Generalized Method of Moments (Advanced Texts in Econometrics). Oxford University Press, Oxford.

Hubalek, F. \& Sgarra, C. (2006). Esscher transforms and the minimal entropy martingale measure for exponential Lévy models. Quantitative Finance, 6(2):125145.

Joe, H. (1989). Relative entropy measures of multivariate dependence. Journal of the American Statistical Association, 84:157-164.

Joe, H. (1997). Multivariate Models and Dependence Concepts, Monographs on Statistics and Applied Probability, volume 73. Chapman \& Hall/CRC, London.

Kallsen, J. \& Tankov, P. (2006). Characterization of dependence of multidimensional Lévy processes using Lévy copulas. Journal of Multivariate Analysis, 97:1551-1572.

Karoui, N. E. \& Quenez, M. (1995). Dynamic programming and pricing of contingent claims in a incomplete market. SIAM Journal of Control and Optimization, 33:29-66.

Karoui, N. E. \& Rouge, R. (2000). Pricing via utility maximization and entropy. Mathematical Finance, 10:259-276.

Kolev, N., Mendes, B. V. M., \& Anjos, U. U. (2005). Copulas: A review and recent developments. Relatório Técnico, IME-USP. São Paulo: RT-MAE 2005-7.

Kou, S. G. (2002). A jump-diffusion model for option pricing. Management Science, 48(8):1086-1101.

Kou, S. G. \& Wang, H. (2004). Option pricing under a double exponential jump difusion model. Management Science, 50(9):1178-1192.

Lindskog, F. \& McNeil, A. J. (2001). Common poisson shock models applications to insurance and credit risk modelling. Artigo em apresentação. Disponível em www.risklab.ch. 
Luciano, E. \& Schoutens, W. (2006). A multivariate jump driven financial asset model. Quatitative Finance, 6(5):385-402.

Madan, D. B., Carr, P. P., \& Chang, E. C. (1990). The variance gamma (V.G.) model for share market returns. Journal of Business, 63:511-524.

Madan, D. B., Carr, P. P., \& Chang, E. C. (1998). The variance gamma processes and option pricing. European Finance Review, 2:79-105.

Maddala, G. S. \& Kim, I. (1999). Unit Roots, Cointegration, and Structural Change. Cambridge, Cambridge.

Matache, A. M., Von Petersdorff, T., \& Schwab, C. (2004). Fast determinisc pricing of options on Lévy driven assets. M2AN Mathematical Modelling and Numerical Analysis, 38:37-71.

Merton, R. C. (1976). Option pricing when the underlying stock returns are discontinuous. Journal of Financial Economics, 3:125-144.

Nelsen, R. B. (1999). An Introduction to Copulas. Springer, New York.

Newey, W. K. \& West, K. D. (1987). A simple, positive semi-definite, heteroskedasticity and autocorrelation consistent covariance matrix. Econometrica, 55(3):703-708.

Prause, K. (1999). The generalized hyperbolic model: Estimation, financial derivatives, and risk measures. Tese de Doutorado - Faculdade de Matemática da Universidade de Freiburg, Alemanha.

Rodriguez, J. C. (2004). Measuring financial contagion: A Copula based approach. EURORANDOM, Report 18.

Rosenberg, J. V. (1999). Semiparametric pricing of multivariate contingent claims. New York. Working paper S.99-35 - Stern School of Business, New York University.

Rosinski, J. (2001). Series representations of lévy processes from the perspective of point proicess. In barndorff-nielsen, o. e.; mikosch, t.; resnick, s. i. (orgs.). Lévy Processes: Theory and applications. Boston: Birkhäuser, 401-415.

Shiryaev, A. N. (1999). Essentials of Stochastic Finance: Facts, Models, Theory. World Scientific, New Jersey.

Taleb, N. (1997). Dynamic Hedging: Managing Vanilla and Exotic Options. John Wiley \& Sons, New York. 


\section{Apêndice}

\section{Extensões de Cópulas Positivas de Lévy aos Casos Multivariados}

Segue um sumário das definições e resultados estendidos aos casos multivariados:

Definição 33 Cópula de Lévy Positiva Uma cópula de Lévy positiva d-dimensional é uma função d-crescente e aderente $C^{\mathcal{L}}:[0 ; \infty]^{d} \longrightarrow[0 ; \infty]$ com marginais $C_{k}^{\mathcal{L}}, k=1,2, \ldots, d$, que satisfazem $C_{k}^{\mathcal{L}}(u)=u$ para todo $u \in[0 ; \infty]$.

Teorema 34 Seja U uma integral caudal de um processo de Lévy com saltos positivos d-dimensional e, seja $U_{1}, \ldots, U_{d}$ as integrais caudais de seus componentes. Existe uma cópula de Lévy positiva d-dimensional $C^{\mathcal{L}}$, tal que, todos os vetores $\left\{x_{1}, \cdots, x_{d}\right\}$ em $\mathbb{R}_{+}^{d}$,

$$
U\left(x_{1}, \cdots, x_{d}\right)=C^{\mathcal{L}}\left(U_{1}\left(x_{1}\right), \cdots, U_{d}\left(x_{d}\right)\right)
$$

Se $U_{1}, \ldots, U_{d}$ são contínuas, então, $C^{\mathcal{L}}$ é unica, caso contrário, é unicamente determinada em $\mathcal{D}\left(C^{\mathcal{L}}\right)=\mathcal{I}\left(U_{1}\right) \times \cdots \times \mathcal{I}\left(U_{d}\right)$. De maneira inversa, se $C^{\mathcal{L}}$ é uma cópula de Lévy positiva d-dimensional e $U_{1}, \ldots, U_{d}$ são as integrais caudais de medidas de Lévy em $[0 ; \infty[$, então, a função $U$ definida acima é uma integral caudal de um processo de Lévy n-dimensional com saltos positivos possuindo marginais $U_{1}, \ldots, U_{d}$.

A cópula de Lévy independente no caso multivariado possui a seguinte forma:

$$
C_{\perp}^{\mathcal{L}}\left(x_{1}, \cdots, x_{d}\right)=x_{1} 1_{\left\{x_{2}=\infty, \ldots, x_{d}=\infty\right\}}+\cdots+x_{d} 1_{\left\{x_{1}=\infty, \ldots, x_{d-1}=\infty\right\}}
$$

e a cópula de Lévy no caso de dependência perfeita é

$$
C_{\|}^{\mathcal{L}}\left(x_{1}, \cdots, x_{d}\right)=\min \left(x_{1}, \cdots, x_{d}\right)
$$
16:

Finalmente, é possível apresentar uma versão multidimensional da Proposição

Proposição 35 Seja $\phi(u)$ uma função estritamente descrescente $\phi:[0 ; \infty] \longrightarrow$ $[0 ; \infty]$, tal que, $\phi(0)=\infty$ e $\phi^{-1}$ possui derivadas de ordem igual a $n$ em $] 0 ; \infty[$ com sinal se alternando com $k$, i.e., $(-1)^{k} \frac{d^{k} \phi^{-1}(t)}{d t^{k}}>0$. Assim,

$$
C^{\mathcal{L}}\left(x_{1}, \cdots, x_{d}\right)=\phi^{-1}\left(\phi\left(x_{1}\right)+\cdots+\phi\left(x_{d}\right)\right)
$$

define uma cópula de Lévy positiva d-dimensional. 


\section{Extensões aos Casos Multivariados de cópulas de Lévy generalizadas}

Damos a seguir um sumário das definições estendidas aos casos multivariados:

Definição 36 Cópula de Lévy Uma cópula de Lévy $n$-dimensional é uma função $C^{\mathcal{L}}: \overline{\mathbb{R}}^{n} \longrightarrow \overline{\mathbb{R}}$ com as seguintes propriedades:

1. $C^{\mathcal{L}}$ é n-crescente;

2. $C^{\mathcal{L}}$ é igual a zero se pelo menos um dos seus argumentos for igual a zero;

3. $C^{\mathcal{L}}$ possui marginais uniformes.

Para construir uma medida de Lévy multidimensional por meio de cópulas de Lévy, é necessário introduzir o seguinte intervalo:

$$
\mathbf{I}(x)=\left\{\begin{array}{rr}
{[x ; \infty[,} & \text { se } x>0 \\
]-\infty ; x], & \text { se } x<0
\end{array}\right.
$$

Ao utilizar esta notação, é possível construir as integrais caudais de uma medida de Lévy em qualquer ponto, salvo naqueles que estão sobre os eixos, como segue:

$$
\begin{aligned}
\nu\left(\mathbf{I}\left(x_{1}\right) \times \cdots \times \mathbf{I}\left(x_{n}\right)\right)= & (-1)^{\left(\operatorname{sgn} x_{1} \times \cdots \times \operatorname{sgn} x_{n}\right)} \\
& C^{\mathcal{L}}\left(U_{1}^{\operatorname{sgn} x_{1}}\left(x_{1}\right), \ldots, U_{n}^{\operatorname{sgn} x_{n}}\left(x_{n}\right)\right)
\end{aligned}
$$

sendo que $U_{1}^{+}, U_{1}^{-}, \ldots, U_{n}^{+}, U_{n}^{-}$são as integrais caudais marginais unidimensionais. $\mathrm{O}$ fato da não unicidade nos eixos pode ser resolvido como foi feito no caso bidimensional. 
Tabela A.1

Análise da influência das cópulas de Lévy de Clayton e Frank sobre os preços de opções asiáticas (venda e compra) considerando médias artiméticas e geométrica

\begin{tabular}{|c|c|c|c|c|c|c|c|c|c|}
\hline \multirow[b]{2}{*}{$\alpha$} & \multirow[b]{2}{*}{ Strike } & \multicolumn{2}{|c|}{$\mathrm{AmC}$} & \multicolumn{2}{|c|}{$\mathrm{AgC}$} & \multicolumn{2}{|c|}{$\mathrm{AmP}$} & \multicolumn{2}{|c|}{ AgP } \\
\hline & & Clayton & Frank & Clayton & Frank & Clayton & Frank & Clayton & Frank \\
\hline \multirow[t]{7}{*}{0} & 20 & 29.1272 & 29.1178 & 29.1223 & 29.1178 & 0 & 0 & 0 & 0 \\
\hline & 30 & 21.8472 & 21.8378 & 21.8423 & 21.8378 & 0 & 0 & 0 & 0 \\
\hline & 40 & 14.5672 & 14.5579 & 14.5624 & 14.5579 & 0 & 0 & 0 & 0 \\
\hline & 50 & 7.2944 & 7.2779 & 7.2902 & 7.2779 & 0.0071 & 0 & 0.0078 & 0 \\
\hline & 60 & 0.1133 & 0 & 0.1103 & 0 & 0.1059 & 0.0021 & 0.1079 & 0.0021 \\
\hline & 70 & 0 & 0 & 0 & 0 & 7.2726 & 7.282 & 7.2775 & 7.282 \\
\hline & 80 & 0 & 0 & 0 & 0 & 14.5526 & 14.562 & 14.5575 & 14.562 \\
\hline \multirow[t]{7}{*}{0.2} & 20 & 26.3975 & 26.39 & 26.2615 & 26.2579 & 0 & 0 & 0 & 0 \\
\hline & 30 & 19.1175 & 19.11 & 18.9815 & 18.9779 & 0 & 0 & 0 & 0 \\
\hline & 40 & 11.8376 & 11.83 & 11.7016 & 11.6979 & 0 & 0 & 0 & 0 \\
\hline & 50 & 4.5675 & 4.5501 & 4.4324 & 4.418 & 0.0099 & 0 & 0.0108 & 0 \\
\hline & 60 & 0.0687 & 0.0626 & 0.0548 & 0.0496 & 2.791 & 2.7925 & 2.9131 & 2.9116 \\
\hline & 70 & 0 & 0 & 0 & 0 & 10.0023 & 10.0098 & 10.1383 & 10.1419 \\
\hline & 80 & 0 & 0 & 0 & 0 & 17.2823 & 17.2898 & 17.4183 & 17.4219 \\
\hline \multirow[t]{7}{*}{$\overline{0.4}$} & 20 & 23.6678 & 23.6621 & 23.4006 & 23.3979 & 0 & 0 & 0 & 0 \\
\hline & 30 & 16.3878 & 16.3822 & 16.1207 & 16.118 & 0 & 0 & 0 & 0 \\
\hline & 40 & 9.1079 & 9.1022 & 8.8407 & 8.838 & 0 & 0 & 0 & 0 \\
\hline & 50 & 2.2706 & 2.2474 & 2.1502 & 2.1299 & 0.4427 & 0.4251 & 0.5895 & 0.5718 \\
\hline & 60 & 0.124 & 0.1253 & 0.0984 & 0.0993 & 5.576 & 5.5829 & 5.8176 & 5.8211 \\
\hline & 70 & 0.0088 & 0.0088 & 0.0048 & 0.0048 & 12.7408 & 12.7464 & 13.0039 & 13.0066 \\
\hline & 80 & 0 & 0 & 0 & 0 & 20.012 & 20.0176 & 20.2791 & 20.2818 \\
\hline \multirow[t]{7}{*}{0.6} & 20 & 20.9381 & 20.9343 & 20.5398 & 20.538 & 0 & 0 & 0 & 0 \\
\hline & 30 & 13.6581 & 13.6544 & 13.2599 & 13.2581 & 0 & 0 & 0 & 0 \\
\hline & 40 & 6.4584 & 6.4565 & 6.2103 & 6.2103 & 0.0802 & 0.0821 & 0.2304 & 0.2322 \\
\hline & 50 & 1.4244 & 1.4183 & 1.3026 & 1.2972 & 2.3262 & 2.3238 & 2.6026 & 2.5991 \\
\hline & 60 & 0.1869 & 0.1879 & 0.1479 & 0.1489 & 8.3686 & 8.3734 & 8.7279 & 8.7307 \\
\hline & 70 & 0.03 & 0.0301 & 0.0181 & 0.0182 & 15.4917 & 15.4955 & 15.8781 & 15.8799 \\
\hline & 80 & 0.0067 & 0.0067 & 0.003 & 0.003 & 22.7484 & 22.7521 & 23.1429 & 23.1447 \\
\hline \multirow[t]{7}{*}{0.8} & 20 & 18.2084 & 18.2065 & 17.679 & 17.6781 & 0 & 0 & 0 & 0 \\
\hline & 30 & 10.9548 & 10.9532 & 10.5845 & 10.584 & 0.0263 & 0.0266 & 0.1855 & 0.1859 \\
\hline & 40 & 4.497 & 4.4968 & 4.2607 & 4.2618 & 0.8485 & 0.8501 & 1.1416 & 1.1436 \\
\hline & 50 & 1.1733 & 1.1724 & 1.045 & 1.0442 & 4.8047 & 4.8058 & 5.2058 & 5.206 \\
\hline & 60 & 0.25 & 0.2505 & 0.198 & 0.1985 & 11.1614 & 11.1638 & 11.6388 & 11.6402 \\
\hline & 70 & 0.0668 & 0.0664 & 0.0443 & 0.0443 & 18.2582 & 18.2596 & 18.7651 & 18.766 \\
\hline & 80 & 0.0176 & 0.0176 & 0.0096 & 0.0096 & 25.4889 & 25.4908 & 26.0103 & 26.0112 \\
\hline \multirow[t]{7}{*}{1} & 20 & 15.4905 & 15.4905 & 14.9759 & 14.9759 & 0.0118 & 0.0118 & 0.1577 & 0.1577 \\
\hline & 30 & 8.5391 & 8.5391 & 8.1784 & 8.1784 & 0.3403 & 0.3403 & 0.6401 & 0.6401 \\
\hline & 40 & 3.409 & 3.409 & 3.1708 & 3.1708 & 2.4902 & 2.4902 & 2.9126 & 2.9126 \\
\hline & 50 & 1.0815 & 1.0815 & 0.9425 & 0.9425 & 7.4426 & 7.4426 & 7.9642 & 7.9642 \\
\hline & 60 & 0.3131 & 0.3131 & 0.2481 & 0.2481 & 13.9542 & 13.9542 & 14.5498 & 14.5498 \\
\hline & 70 & 0.1123 & 0.1123 & 0.0786 & 0.0786 & 21.0334 & 21.0334 & 21.6602 & 21.6602 \\
\hline & 80 & 0.0343 & 0.0343 & 0.0204 & 0.0204 & 28.2354 & 28.2354 & 28.8819 & 28.8819 \\
\hline
\end{tabular}


Tabela A.2

Análise da influência da dependência (linear) sobre o preço de opções asiáticas (compra e venda) considerando a média aritmética e a cópula de Lévy de Clayton

\begin{tabular}{|c|c|c|c|c|c|c|c|}
\hline & & & AmC & & & $\mathbf{A m P}$ & \\
\hline$\alpha$ & Strike / $\rho$ & 0.187 & 0.0943 & 0.0482 & 0.187 & 0.0943 & 0.0482 \\
\hline \multirow[t]{7}{*}{0} & 20 & 30.2216 & 30.4886 & 30.7076 & 0 & 0 & 0.0009 \\
\hline & 30 & 22.9622 & 23.2132 & 23.4386 & 0.0205 & 0.0045 & 0.0118 \\
\hline & 40 & 15.9056 & 16.1152 & 16.2451 & 0.2439 & 0.1865 & 0.0983 \\
\hline & 50 & 9.6632 & 9.8058 & 9.6717 & 1.2814 & 1.1571 & 0.8049 \\
\hline & 60 & 5.186 & 5.1585 & 4.7653 & 4.0843 & 3.7897 & 3.1784 \\
\hline & 70 & 2.5303 & 2.4476 & 2.0408 & 8.7085 & 8.3588 & 7.7338 \\
\hline & 80 & 1.1742 & 1.1361 & 0.8775 & 14.6323 & 14.3272 & 13.8505 \\
\hline \multirow[t]{7}{*}{0.2} & 20 & 27.2313 & 27.5794 & 27.6216 & 0 & 0 & 0 \\
\hline & 30 & 19.9689 & 20.3037 & 20.3484 & 0.0175 & 0.0042 & 0.0068 \\
\hline & 40 & 12.934 & 13.2025 & 13.1556 & 0.2626 & 0.183 & 0.0939 \\
\hline & 50 & 7.0536 & 7.1098 & 6.8331 & 1.6621 & 1.3702 & 1.0514 \\
\hline & 60 & 3.245 & 3.143 & 2.7405 & 5.1335 & 4.6834 & 4.2388 \\
\hline & 70 & 1.3336 & 1.2596 & 0.9581 & 10.502 & 10.08 & 9.7363 \\
\hline & 80 & 0.541 & 0.5058 & 0.3654 & 16.9894 & 16.6061 & 16.4236 \\
\hline \multirow[t]{7}{*}{0.4} & 20 & 24.2411 & 24.6702 & 24.5364 & 0 & 0 & 0 \\
\hline & 30 & 16.9792 & 17.3989 & 17.2592 & 0.0181 & 0.0086 & 0.0028 \\
\hline & 40 & 10.0402 & 10.3494 & 10.1225 & 0.359 & 0.2391 & 0.146 \\
\hline & 50 & 4.7369 & 4.6989 & 4.3318 & 2.3356 & 1.8685 & 1.6352 \\
\hline & 60 & 1.751 & 1.6584 & 1.3534 & 6.6297 & 6.108 & 5.9368 \\
\hline & 70 & 0.5947 & 0.5507 & 0.3775 & 12.7534 & 12.2803 & 12.2409 \\
\hline & 80 & 0.1815 & 0.1476 & 0.125 & 19.6201 & 19.1572 & 19.2683 \\
\hline \multirow[t]{7}{*}{0.6} & 20 & 21.2509 & 21.761 & 21.4512 & 0 & 0 & 0 \\
\hline & 30 & 14.0071 & 14.4966 & 14.179 & 0.0362 & 0.0156 & 0.0078 \\
\hline & 40 & 7.3431 & 7.6338 & 7.2608 & 0.6521 & 0.4327 & 0.3695 \\
\hline & 50 & 2.8697 & 2.8377 & 2.4572 & 3.4587 & 2.9165 & 2.8458 \\
\hline & 60 & 0.8527 & 0.7905 & 0.6382 & 8.7216 & 8.1493 & 8.3068 \\
\hline & 70 & 0.2224 & 0.1927 & 0.1523 & 15.3713 & 14.8315 & 15.1009 \\
\hline & 80 & 0.0443 & 0.0441 & 0.05 & 22.4731 & 21.9628 & 22.2786 \\
\hline \multirow[t]{7}{*}{0.8} & 20 & 18.2615 & 18.8518 & 18.366 & 0.0008 & 0 & 0 \\
\hline & 30 & 11.0871 & 11.6352 & 11.1442 & 0.1064 & 0.0633 & 0.0581 \\
\hline & 40 & 5.0073 & 5.3085 & 4.848 & 1.3066 & 1.0166 & 1.0419 \\
\hline & 50 & 1.6736 & 1.688 & 1.4378 & 5.2529 & 4.676 & 4.9116 \\
\hline & 60 & 0.451 & 0.445 & 0.4029 & 11.3102 & 10.7131 & 11.1567 \\
\hline & 70 & 0.1013 & 0.1071 & 0.1056 & 18.2405 & 17.6551 & 18.1394 \\
\hline & 80 & 0.0078 & 0.0303 & 0.0271 & 25.4269 & 24.8583 & 25.3408 \\
\hline \multirow[t]{7}{*}{1} & 20 & 15.2919 & 15.9581 & 15.3003 & 0.0215 & 0.0155 & 0.0194 \\
\hline & 30 & 8.3882 & 9.0045 & 8.405 & 0.3978 & 0.3418 & 0.4041 \\
\hline & 40 & 3.3621 & 3.7285 & 3.3253 & 2.6517 & 2.3459 & 2.6043 \\
\hline & 50 & 1.1278 & 1.2336 & 1.085 & 7.6973 & 7.1309 & 7.644 \\
\hline & 60 & 0.3393 & 0.3883 & 0.3684 & 14.1887 & 13.5655 & 14.2073 \\
\hline & 70 & 0.0741 & 0.109 & 0.1195 & 21.2035 & 20.5662 & 21.2384 \\
\hline & 80 & 0.0062 & 0.0255 & 0.0433 & 28.4155 & 27.7627 & 28.4422 \\
\hline
\end{tabular}


Tabela A.3

Análise da influência da dependência (linear) sobre o preço de opções asiáticas (compra e venda) considerando a média aritimética e a cópula de Lévy de Frank

\begin{tabular}{|c|c|c|c|c|c|c|c|}
\hline & & & $\mathrm{AmC}$ & & & $\mathrm{AmP}$ & \\
\hline$\alpha$ & Strike $\rho$ & 0.1259 & 0.1068 & 0.0741 & 0.1259 & 0.1068 & 0.0741 \\
\hline \multirow[t]{7}{*}{0} & 20 & 32.1586 & 32.7549 & 36.6994 & 0.0041 & 0.0013 & 0.0387 \\
\hline & 30 & 24.977 & 25.6092 & 29.824 & 0.1025 & 0.1356 & 0.4433 \\
\hline & 40 & 18.3596 & 19.0198 & 23.6615 & 0.765 & 0.8261 & 1.5607 \\
\hline & 50 & 12.8714 & 13.4749 & 18.4543 & 2.5567 & 2.5612 & 3.6335 \\
\hline & 60 & 8.7148 & 9.1665 & 14.2188 & 5.6801 & 5.5328 & 6.6779 \\
\hline & 70 & 5.711 & 6.1052 & 10.8583 & 9.9563 & 9.7515 & 10.5973 \\
\hline & 80 & 3.6876 & 4.0198 & 8.2757 & 15.2128 & 14.946 & 15.2947 \\
\hline \multirow[t]{7}{*}{0.2} & 20 & 28.7777 & 29.3913 & 32.3945 & 0 & 0 & 0.0098 \\
\hline & 30 & 21.5626 & 22.1944 & 25.3658 & 0.0649 & 0.083 & 0.261 \\
\hline & 40 & 14.9378 & 15.5583 & 19.114 & 0.72 & 0.7268 & 1.2892 \\
\hline & 50 & 9.6596 & 10.1849 & 14.0518 & 2.7218 & 2.6334 & 3.5069 \\
\hline & 60 & 5.93 & 6.3276 & 10.1315 & 6.2722 & 6.056 & 6.8665 \\
\hline & 70 & 3.4866 & 3.8035 & 7.2442 & 11.1087 & 10.8119 & 11.2592 \\
\hline & 80 & 2.0066 & 2.2586 & 5.1765 & 16.9087 & 16.547 & 16.4714 \\
\hline \multirow[t]{7}{*}{0.4} & 20 & 25.4008 & 26.0292 & 28.1109 & 0 & 0 & 0.0021 \\
\hline & 30 & 18.1736 & 18.8099 & 20.9816 & 0.0527 & 0.0607 & 0.1527 \\
\hline & 40 & 11.5988 & 12.1563 & 14.6581 & 0.7579 & 0.687 & 1.1093 \\
\hline & 50 & 6.6259 & 7.1084 & 9.7925 & 3.065 & 2.9191 & 3.5236 \\
\hline & 60 & 3.5049 & 3.8174 & 6.3629 & 7.2239 & 6.908 & 7.3739 \\
\hline & 70 & 1.7264 & 1.9676 & 4.0943 & 12.7253 & 12.3383 & 12.3853 \\
\hline & 80 & 0.7961 & 0.996 & 2.5822 & 19.075 & 18.6466 & 18.1532 \\
\hline \multirow[t]{7}{*}{0.6} & 20 & 22.0247 & 22.667 & 23.8331 & 0.0007 & 0 & 0.0003 \\
\hline & 30 & 14.8179 & 15.4451 & 16.6603 & 0.0739 & 0.0581 & 0.1074 \\
\hline & 40 & 8.3947 & 8.9204 & 10.348 & 0.9306 & 0.8134 & 1.0752 \\
\hline & 50 & 3.9692 & 4.3549 & 5.8393 & 3.785 & 3.5278 & 3.8464 \\
\hline & 60 & 1.6299 & 1.8564 & 3.1523 & 8.7257 & 8.3093 & 8.4393 \\
\hline & 70 & 0.5841 & 0.7355 & 1.6387 & 14.9599 & 14.4683 & 14.2057 \\
\hline & 80 & 0.1719 & 0.2934 & 0.8893 & 21.8276 & 21.3062 & 20.7362 \\
\hline \multirow[t]{7}{*}{0.8} & 20 & 18.6526 & 19.3064 & 19.5571 & 0.0054 & 0.0017 & 0.0003 \\
\hline & 30 & 11.5127 & 12.1276 & 12.4071 & 0.1454 & 0.1028 & 0.1302 \\
\hline & 40 & 5.4779 & 5.954 & 6.3275 & 1.3906 & 1.2091 & 1.3306 \\
\hline & 50 & 1.9897 & 2.2404 & 2.6267 & 5.1824 & 4.7755 & 4.9098 \\
\hline & 60 & 0.6039 & 0.7148 & 0.9946 & 11.0765 & 10.5299 & 10.5576 \\
\hline & 70 & 0.1338 & 0.1944 & 0.3688 & 17.8864 & 17.2894 & 17.2118 \\
\hline & 80 & 0.015 & 0.0451 & 0.1694 & 25.0475 & 24.4201 & 24.2923 \\
\hline \multirow[t]{7}{*}{1} & 20 & 15.2919 & 15.9581 & 15.3003 & 0.0215 & 0.0155 & 0.0194 \\
\hline & 30 & 8.3882 & 9.0045 & 8.405 & 0.3978 & 0.3418 & 0.4041 \\
\hline & 40 & 3.3621 & 3.7285 & 3.3253 & 2.6517 & 2.3459 & 2.6043 \\
\hline & 50 & 1.1278 & 1.2336 & 1.085 & 7.6973 & 7.1309 & 7.644 \\
\hline & 60 & 0.3393 & 0.3883 & 0.3684 & 14.1887 & 13.5655 & 14.2073 \\
\hline & 70 & 0.0741 & 0.109 & 0.1195 & 21.2035 & 20.5662 & 21.2384 \\
\hline & 80 & 0.0062 & 0.0255 & 0.0433 & 28.4155 & 27.7627 & 28.4422 \\
\hline
\end{tabular}

